\title{
Perturbation theory without diagrams: The polaron case
}

\author{
R. Rosenfelder* \\ Particle Theory Group, Paul Scherrer Institute, CH-5232 Villigen PSI, Switzerland
}

(Received 9 July 2008; published 13 January 2009)

\begin{abstract}
Higher-order perturbative calculations in quantum (field) theory suffer from the factorial increase of the number of individual diagrams. Here I describe an approach which evaluates the total contribution numerically for finite temperature from the cumulant expansion of the corresponding observable followed by an extrapolation to zero temperature. This method (originally proposed by Bogolyubov and Plechko) is applied to the calculation of higher-order terms for the ground-state energy of the polaron. Using state-of-the-art multidimensional integration routines two new coefficients are obtained corresponding to a four- and five-loop calculation. Several analytical and numerical procedures have been implemented which were crucial for obtaining reliable results.
\end{abstract}

DOI: 10.1103/PhysRevE.79.016705

PACS number(s): 02.70.Uu, 11.15.Bt, 71.38.Fp

\section{INTRODUCTION}

Highly accurate measurements require precise theoretical calculations which perturbation theory can yield if the coupling constant is small. However, in quantum field theory the number of diagrams grows factorially with the order of perturbation theory and they become more and more complicated as the corresponding loop diagrams involve highdimensional integrals over complicated (and singular) functions.

The prime example is the anomalous magnetic moment of the electron where new experiments [1,2] need high-order quantum-electrodynamical calculations. In fact, the estimate for the fifth-order contribution is the largest source of theoretical uncertainty if one attributes an "error" to it at all [3]. In addition, further improvements of the experimental accuracy are foreseen.

As derived in the textbook [4] the number of diagrams contributing to the vertex function in quantum electrodynamics (QED) is given by the coefficients of the generating function

$$
\Gamma=\frac{4 z(1-S)}{S^{3}}, \quad S=-2 z\left[1+\frac{K_{0}^{\prime}(z)}{K_{0}(z)}\right]
$$

[with $z=-1 /(4 \alpha)$ and $K_{0}(z)$ the zeroth-order modified Bessel function of second kind] when expanded in powers of the fine-structure constant $\alpha$

$$
\begin{aligned}
\Gamma(\alpha)= & 1+\alpha+7 \alpha^{2}+72 \alpha^{3}+891 \alpha^{4}+12672 \alpha^{5}+202770 \alpha^{6} \\
& +\cdots
\end{aligned}
$$

The contributions up to third order are known analytically [5] and the 891 diagrams in fourth order have been evaluated numerically by Kinoshita and co-workers [6]. In view of the ever more precise experiments there are ongoing efforts [7] to calculate all 12672 diagrams in $O\left(\alpha^{5}\right)$ numerically and by automated routines. This is a huge, heroic effort considering the complexity of individual diagrams, the large cancella-

\footnotetext{
*roland.rosenfelder@psi.ch
}

tions among them and the intricacies of infrared and ultraviolet divergencies in the integrands.

Obviously new and more efficient methods would be most welcome for a cross-check as well as further progress. However, it is useful first to consider a simpler field theory which is nontrivial but free from ultraviolet divergencies. This is supplied by the polaron problem - the field theory of a single nonrelativistic electron slowly moving in a polarizable crystal and thereby interacting with an infinite number of phonons. Similar as in quantum electrodynamics there exists a large number of perturbative calculations for the groundstate energy and other properties of the quasiparticle which is made up by the electron and its surrounding cloud of virtual phonons.

In this paper we investigate a method originally proposed by Bogolyubov (Jr.) and Plechko (BP) [8] to obtain higherorder terms in the ground-state energy of a polaron without evaluating diagrams. As the polaron problem is the prototype of the worldline approach to relativistic quantum field theory [9-12] we believe that a similar method also holds promise for high-order perturbative calculations in particle physics, in particular QED.

Preliminary results have already been presented in Ref. [13]. Here I give a detailed account of the analytical and numerical methods which are required so that the BP method works. The paper is organized as follows. In Secs. II and III we recall the basics of the polaron model and the BP method. Section IV gives an account of the necessary steps to obtain reliable numerical results. These are presented and discussed in Sec. V. The last section contains our conclusion and the outlook for further work whereas more technical details are collected in three appendixes.

\section{THE POLARON PROBLEM-A NONRELATIVISTIC FIELD THEORY}

A model Hamiltonian describing the dressing of the bare electron by a cloud of phonons has been given by Fröhlich

$$
\begin{aligned}
\hat{H}= & \frac{1}{2} \hat{\mathbf{p}}^{2}+\int d^{3} k \hat{a}_{\mathbf{k}}^{\dagger} \hat{a}_{\mathbf{k}}+i(2 \sqrt{2} \pi \alpha)^{1 / 2} \int \frac{d^{3} k}{(2 \pi)^{3}} \frac{1}{|\mathbf{k}|}\left[\hat{a}_{\mathbf{k}}^{\dagger} e^{i \mathbf{k} \cdot \hat{\mathbf{x}}}\right. \\
& - \text { H.c. }], \quad\left[\hat{a}_{\mathbf{k}}, \hat{a}_{\mathbf{k}^{\prime}}^{\dagger}\right]=\delta^{(3)}\left(\mathbf{k}-\mathbf{k}^{\prime}\right),
\end{aligned}
$$

where $\alpha$ is the dimensionless electron-phonon coupling con- 
stant. Due to its interaction with the medium the energy of the quasiparticle is changed and it acquires an effective mass

$$
E_{\mathbf{p}}=E_{0}+\frac{\mathbf{p}^{2}}{2 m^{\star}}+\cdots \text {. }
$$

The aim is to calculate the power series expansion for the ground-state energy of a nonmoving polaron

$$
E_{0}(\alpha)=: \sum_{n=1} e_{n} \alpha^{n}
$$

as function of $\alpha$ [14]. The lowest-order coefficients are well known

$$
\begin{gathered}
e_{1}=-1, \\
e_{2}=\frac{1}{\sqrt{2}}-\ln \left(1+\frac{3}{4} \sqrt{2}\right)=-0.015919622
\end{gathered}
$$

from Ref. [15],

$$
e_{3}=-0.000806070
$$

from Refs. $[16,17]$, but there has been no progress towards higher-order terms.

In the path-integral approach [18] the (infinite) phonon degrees of freedom may be integrated out exactly which leads to an effective, two-time action

$$
\begin{aligned}
S[\mathbf{x}]= & \int_{0}^{\beta} d t \frac{1}{2} \dot{\mathbf{x}}^{2}-\frac{\alpha}{2 \sqrt{2}} \int_{0}^{\beta} d t d t^{\prime} \\
& \times \frac{\left.\cosh \left[\beta / 2-\left|t-t^{\prime}\right|\right)\right]}{\sinh (\beta / 2)} \frac{1}{\left|\mathbf{x}(t)-\mathbf{x}\left(t^{\prime}\right)\right|} .
\end{aligned}
$$

Here $\beta$ is the Euclidean time or inverse temperature. Some simplifications are possible: first, the symmetry between the two times $t, t^{\prime}$ allows us to restrict the integration range of the latter to $0 \leqslant t^{\prime} \leqslant t$ together with doubling the strength of the interaction. Second, as we are only interested in the ground-state energy $E_{0}$ of the polaron which can be obtained by the large- $\beta$ limit of the partition function

$$
Z:=\int d^{3} x \int_{\mathbf{x}(0)=\mathbf{x}(\beta)=\mathbf{x}} \mathcal{D}^{3} x e^{-S[\mathbf{x}]} \rightarrow \operatorname{const} e^{-\beta \mathrm{E}_{0},}
$$

we may replace

$$
\begin{aligned}
& \frac{\cosh \left[\beta / 2-\left(t-t^{\prime}\right)\right]}{\sinh (\beta / 2)} \\
& \quad=\frac{\exp (-\sigma)+\exp [-(\beta-\sigma)]^{\beta \rightarrow \infty}}{1-\exp (-\beta)} \rightarrow \exp (-\sigma),
\end{aligned}
$$

where $\sigma=t-t^{\prime}$ is the relative time [19]. Thus, in the following, we will use

$$
S[\mathbf{x}]=\int_{0}^{\beta} d t \frac{1}{2} \dot{\mathbf{x}}^{2}-\frac{\alpha}{\sqrt{2}} \int_{0}^{\beta} d t \int_{0}^{t} d t^{\prime} \frac{\exp (-\sigma)}{\left|\mathbf{x}(t)-\mathbf{x}\left(t^{\prime}\right)\right|}=: S_{0}+S_{1}
$$

as a full polaron action.
Useful order-of-magnitude estimates for higher-order energy coefficients can be obtained in various approximate treatments of the polaron problem. Most prominent and successful among these is Feynman's approach [18] in which a quadratic trial action

$$
S_{t}=\int_{0}^{\beta} d t \frac{1}{2} \dot{\mathbf{x}}^{2}+\int_{0}^{\beta} d t \int_{0}^{t} d t^{\prime} f\left(t-t^{\prime}\right)\left[\mathbf{x}(t)-\mathbf{x}\left(t^{\prime}\right)\right]^{2}
$$

is used as variational approximation for the full action (12). Feynman chose an exponential form of the retardation function with two variational parameters which are determined by minimizing Jensen's inequality. The corresponding energy coefficients can be calculated analytically to high order [17] as sketched in Appendix A. The result is

$$
\begin{gathered}
e_{1}^{F}=-1, \quad e_{2}^{F}=-\frac{1}{81}=-1.234568 \times 10^{-2}, \\
e_{3}^{F}=\frac{16}{729}-\frac{56}{6561} \sqrt{7}=-0.634366 \times 10^{-3},
\end{gathered}
$$

$$
e_{4}^{F}=\frac{3200 \sqrt{10}-633236}{1594323}+\frac{78496}{531441} \sqrt{7}=-0.464315 \times 10^{-4}
$$

$$
\begin{aligned}
e_{5}^{F}= & \frac{1673496632-6044800 \sqrt{10}-70304 \sqrt{13}}{129140163} \\
& +\frac{793600}{43046721} \sqrt{70}-\frac{1476371144}{301327047} \sqrt{7}=-0.395686 \times 10^{-5} .
\end{aligned}
$$

However, one can do better by allowing the variational principle to determine the best retardation function itself. Then one gets $[20,21]$

$$
e_{1}^{\text {best }}=-1, \quad e_{2}^{\text {best }}=-\left(\frac{1}{12}-\frac{2}{9 \pi}\right)=-1.2597803 \times 10^{-2} .
$$

Note that $e_{2}^{\text {best }}$ is only slightly better than $e_{2}^{F}$ despite the fact that the retardation function in the unrestricted variational approach has quite a different small-time behavior than Feynman's parametrization. This is due to the (relative) insensitivity of the polaron energy to small-time dynamics. In this respect four-dimensional field theories in the worldline description are quite different, in particular realistic, renormalizable ones similar to QED [12]. Appendix A also describes how one can obtain numerically the higher-order energy coefficients for the best quadratic approximation. We have obtained the values

$$
\begin{gathered}
e_{3}^{\text {best }}=-0.64650 \times 10^{-3}, \quad e_{4}^{\text {best }}=-0.4686 \times 10^{-4}, \\
e_{5}^{\text {best }}=-0.3940 \times 10^{-5}
\end{gathered}
$$

which-again_-are not very much different from the results using the much simpler Feynman parametrization. 


\section{THE BOGOLIUBOV-PLECHKO (BP) METHOD}

In order to get the perturbative expansion of $E_{0}(\alpha)$ we use the cumulant expansion of the partition function for large $\beta$

$$
Z=Z_{0} \exp \left[\sum_{n=1} \frac{(-)^{n}}{n !} \lambda_{n}(\beta)\right]
$$

where $\lambda_{n}(\beta)$ are the cumulants with respect to $S_{1}$ and $Z_{0}$ is the free partition function for a system confined in a large volume.

The cumulants (or semi-invariants) are obtained from the (normalized) moments

$$
m_{n} \equiv\left\langle S_{1}^{n}\right\rangle:=C \int d^{3} x \int_{\mathbf{x}(0)=\mathbf{x}}^{\mathbf{x}(\beta)=\mathbf{x}} \mathcal{D}^{3} x S_{1}^{n} e^{-S_{0}[\mathbf{x}]}
$$

(here the $\beta$ dependence is suppressed and the normalization constant $C$ is chosen such that $m_{0}=1$ ) via the recursion relation

$$
\lambda_{n+1}=m_{n+1}-\sum_{k=0}^{n-1}\left(\begin{array}{l}
n \\
k
\end{array}\right) \lambda_{k+1} m_{n-k} .
$$

This is standard and easily proved by differentiating the characteristic function [22]

$$
\Phi(t)=\left\langle e^{-t S_{1}}\right\rangle=\sum_{n=0}(-)^{n} \frac{t^{n}}{n !} m_{n}=\exp \left[\sum_{n=1}(-)^{n} \frac{t^{n}}{n !} \lambda_{n}\right]
$$

with respect to $t$ in moment and cumulant form

$$
-\sum_{n=0} \frac{(-t)^{n}}{n !} m_{n+1}=-\Phi(t) \sum_{n=0} \frac{(-t)^{n}}{n !} \lambda_{n+1} .
$$

If the moment expansion for $\Phi(t)$ is inserted on the righthand size one obtains after rearrangement

$$
-\sum_{n=0} \frac{(-t)^{n}}{n !} m_{n+1}=-\sum_{n=0}(-t)^{n} \sum_{k=0}^{n} \frac{1}{k !(n-k) !} \lambda_{k+1} m_{n-k}
$$

for all powers of $t$ which establishes Eq. (20). The first few cumulants are

$$
\begin{gathered}
\lambda_{1}=m_{1} \\
\lambda_{2}=m_{2}-m_{1}^{2} \\
\lambda_{3}=m_{3}-3 m_{2} m_{1}+2 m_{1}^{3} \\
\lambda_{4}=m_{4}-4 m_{3} m_{1}-3 m_{2}^{2}+12 m_{2} m_{1}^{2}-6 m_{1}^{4} \\
\lambda_{5}=m_{5}-5 m_{4} m_{1}-10 m_{3} m_{2}+20 m_{3} m_{1}^{2}+30 m_{2}^{2} m_{1}-60 m_{2} m_{1}^{3} \\
+24 m_{1}^{5}
\end{gathered}
$$

For large $\beta$ we then get the ground-state energy as zerotemperature limit of the free energy

$$
E_{0}=\lim _{\beta \rightarrow \infty}\left(-\frac{1}{\beta}\right) \sum_{n=1} \frac{(-)^{n}}{n !} \lambda_{n}(\beta)
$$

since the free partition function does not contribute. By construction the $n$th moment is proportional to $\alpha^{n}$ and Eq. (20) (and the examples) show that the cumulants share this properties. Comparing with Eq. (5) we see that

$$
e_{n}=\frac{(-)^{n+1}}{\alpha^{n} n !} \lim _{\beta \rightarrow \infty} \frac{1}{\beta} \lambda_{n}(\beta) .
$$

The moments $m_{n}$. We calculate the moments $m_{n}$ by expanding the paths in Fourier components

$$
\mathbf{x}(t)=\sqrt{2 \beta} \mathbf{b}_{0} \frac{t}{\beta}+\sum_{k=1}^{\infty} \frac{2 \sqrt{\beta}}{k \pi} \mathbf{b}_{k} \sin \left(\frac{k \pi t}{\beta}\right), \quad \mathbf{x}=: \sqrt{2 \beta} \mathbf{b}_{0}
$$

so that

$$
S_{0}=\sum_{k=0}^{\infty} \mathbf{b}_{k}^{2}
$$

and the functional integration is over the coefficients $\mathbf{b}_{k}$, $k=0,1, \ldots$. Writing

$$
S_{1}=-\frac{\alpha}{\sqrt{2}} \int_{0}^{\beta} d t \int_{0}^{t} d t^{\prime} e^{-\left(t-t^{\prime}\right)} \int \frac{d^{3} p}{2 \pi^{2}} \frac{1}{\mathbf{p}^{2}} \exp \left\{i \mathbf{p} \cdot\left[\mathbf{x}(t)-\mathbf{x}\left(t^{\prime}\right)\right]\right\}
$$

we have

$$
\begin{aligned}
m_{n}= & (-)^{n} \frac{\alpha^{n}}{2^{n / 2}} \int_{0}^{\beta} d t_{1} \cdots d t_{n} \int_{0}^{t_{1}} d t_{1}^{\prime} \cdots \int_{0}^{t_{n}} d t_{n}^{\prime} \\
& \times \exp \left[-\left(t_{1}-t_{1}^{\prime}\right)-\cdots-\left(t_{n}-t_{n}^{\prime}\right)\right] \\
& \times \int \frac{d^{3} p_{1}}{2 \pi^{2}} \frac{1}{\mathbf{p}_{1}^{2}} \cdots \int \frac{d^{3} p_{n}}{2 \pi^{2}} \frac{1}{\mathbf{p}_{n}^{2}} \\
& \times\left\langle\exp \left[2 i \sum_{m=1}^{n} \mathbf{p}_{m} \cdot \sum_{k=0}^{\infty} l_{k}\left(t_{m}, t_{m}^{\prime}\right) \mathbf{b}_{k}\right]\right\rangle,
\end{aligned}
$$

where

$$
l_{k}\left(t, t^{\prime}\right)= \begin{cases}\frac{1}{\sqrt{2 \beta}}\left(t-t^{\prime}\right): & k=0, \\ \frac{\sqrt{\beta}}{k \pi}\left(\sin \frac{k \pi t}{\beta}-\sin \frac{k \pi t^{\prime}}{\beta}\right): & k \geqslant 1\end{cases}
$$

and

$$
\langle\mathcal{O}\rangle:=\frac{\int d^{3} b_{0} d^{3} b_{1} \cdots \mathcal{O}\left(\mathbf{b}_{0}, \mathbf{b}_{1}, \ldots\right) \exp \left[-S_{0}\left(\mathbf{b}_{0}, \mathbf{b}_{1}, \ldots\right)\right]}{\int d^{3} b_{0} d^{3} b_{1} \cdots \exp \left[-S_{0}\left(\mathbf{b}_{0}, \mathbf{b}_{1}, \ldots\right)\right]}
$$

is the average with respect to the free action $S_{0}$.

As a Gaussian integral over the $\mathbf{b}_{k}$ 's this average can be done easily and one obtains 


$$
\begin{aligned}
m_{n}= & (-)^{n} \frac{\alpha^{n}}{2^{n / 2}} \prod_{m=1}^{n}\left(\int_{0}^{\beta} d t_{m} \int_{0}^{t_{m}} d t_{m}^{\prime}\right) \exp \left[-\sum_{m=1}^{n}\left(t_{m}-t_{m}^{\prime}\right)\right] \\
& \times \prod_{m=1}^{n}\left(\int \frac{d^{3} p_{m}}{2 \pi^{2}} \frac{1}{\mathbf{p}_{m}^{2}}\right) \exp \left[-\sum_{k=0}^{\infty}\left(\sum_{m=1}^{n} l_{k}\left(t_{m}, t_{m}^{\prime}\right) \mathbf{p}_{m}\right)^{2}\right]
\end{aligned}
$$

If we now write the $m$ th Coulomb propagator as

$$
\frac{1}{\mathbf{p}_{m}^{2}}=\frac{1}{2} \int_{0}^{\infty} d u_{m} \exp \left[-\frac{1}{2} \mathbf{p}_{m}^{2} u_{m}\right]
$$

then all momentum integrations can be performed and give the result

$$
\begin{aligned}
m_{n}= & (-)^{n} \frac{\alpha^{n}}{(4 \pi)^{n / 2}} \prod_{m=1}^{n}\left(\int_{0}^{\beta} d t_{m} \int_{0}^{t_{m}} d t_{m}^{\prime} \int_{0}^{\infty} d u_{m}\right) \\
& \times \exp \left[-\sum_{m=1}^{n}\left(t_{m}-t_{m}^{\prime}\right)\right] \\
& \times\left[\operatorname{det}_{n} A\left(t_{1}, \ldots, t_{n} ; t_{1}^{\prime}, \ldots, t_{n}^{\prime} ; u_{1}, \ldots, u_{n}\right)\right]^{-3 / 2} .
\end{aligned}
$$

Here $(A)$ is the $n \times n$ matrix made up by the elements

$$
(A)_{i j}=2 \sum_{k=0}^{\infty} l_{k}\left(t_{i}, t_{i}^{\prime}\right) l_{k}\left(t_{j}, t_{j}^{\prime}\right)+u_{i} \delta_{i j}=: a_{i j}+u_{i} \delta_{i j} .
$$

It is essential that the infinite sum over the modes $k$ can be performed analytically. Using Eq. (1.443.3) in Ref. [23]

$$
\sum_{k=1}^{\infty} \frac{\cos k x}{k^{2}}=\frac{\pi^{2}}{6}-\frac{\pi x}{2}+\frac{x^{2}}{4}, \quad 0 \leqslant x \leqslant 2 \pi
$$

we indeed have

$$
\begin{aligned}
\sum_{k=1}^{\infty} & \frac{1}{k^{2} \pi^{2}} \sin \frac{k \pi x}{\beta} \sin \frac{k \pi y}{\beta} \\
\quad= & \frac{1}{2} \sum_{k=1}^{\infty} \frac{1}{k^{2} \pi^{2}}\left[\cos \frac{k \pi(x-y)}{\beta}-\cos \frac{k \pi(x+y)}{\beta}\right] \\
& =\frac{1}{2 \beta}\left[\min (x, y)-\frac{x y}{\beta}\right], \quad 0 \leqslant x, y \leqslant \beta
\end{aligned}
$$

and, therefore,

$$
a_{i j}=\min \left(t_{i}, t_{j}\right)-\min \left(t_{i}, t_{j}^{\prime}\right)-\min \left(t_{i}^{\prime}, t_{j}\right)+\min \left(t_{i}^{\prime}, t_{j}^{\prime}\right) .
$$

Using $\min (x, y)=[x+y-|x-y|] / 2$ this may also be written as

$$
a_{i j}=\frac{1}{2}\left[-\left|t_{i}-t_{j}\right|+\left|t_{i}-t_{j}^{\prime}\right|+\left|t_{i}^{\prime}-t_{j}\right|-\left|t_{i}^{\prime}-t_{j}^{\prime}\right|\right] .
$$

Note that $a_{i j}=a_{j i}$ and that

$$
a_{i i}=t_{i}-t_{i}^{\prime}=: \sigma_{i} \geqslant 0
$$

since $t_{i} \geqslant t_{i}^{\prime}$. This is a special case of the more general fact that $(A)$ is a positive definite matrix (otherwise the momen-

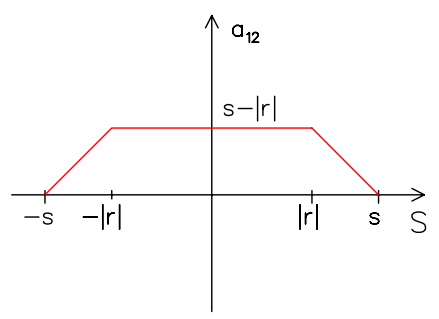

FIG. 1. (Color online) The nondiagonal matrix element $a_{12}$ of the matrix $(A)$ as a function of the time variables defined in Eq. (48).

tum integral would not converge) [24]. Well-known theorems of matrix analysis $[25,26]$ then guarantee that the principal minors of all orders are non-negative and the diagonal elements are just the ones of lowest order.

Introducing total and relative times

$$
\sigma_{i}:=t_{i}-t_{i}^{\prime}, \quad \Sigma_{i}:=\frac{t_{i}+t_{i}^{\prime}}{2}
$$

we have

$$
\begin{aligned}
m_{n}= & (-)^{n} \frac{\alpha^{n}}{(4 \pi)^{n / 2}} \prod_{m=1}^{n}\left(\int_{0}^{\beta} d \sigma_{m} \int_{\sigma_{m} / 2}^{\beta-\sigma_{m} / 2} d \Sigma_{m} \int_{0}^{\infty} d u_{m}\right) \\
& \times \exp \left[-\sum_{m=1}^{n} \sigma_{m}\right] \\
& \times\left[\operatorname{det}_{n} A\left(\sigma_{1}, \ldots, \sigma_{n}, \Sigma_{1}, \ldots, \Sigma_{n} ; u_{1}, \ldots, u_{n}\right)\right]^{-3 / 2}
\end{aligned}
$$

Due to time-translational invariance, the nondiagonal matrix elements, say $a_{12}$, only depend on three variables which we denote by

$$
S:=\Sigma_{1}-\Sigma_{2}, \quad r:=\frac{1}{2}\left(\sigma_{1}-\sigma_{2}\right), \quad s:=\frac{1}{2}\left(\sigma_{1}+\sigma_{2}\right) \geqslant 0 .
$$

Then one has

$$
\begin{aligned}
a_{12}= & \frac{1}{2}[|S+s|+|S-s|-|S+r|-|S-r|] \\
= & \begin{cases}s-|r| & \text { for }|S| \leqslant|r|, \\
s-|S| & \text { for }|r| \leqslant|S| \leqslant s, \\
0 & \text { for }|S| \geqslant s .\end{cases}
\end{aligned}
$$

Figure 1 shows that $a_{12}$ is indeed a nonanalytic function of the times as expected from the absolute values in Eq. (44). Note that it is even in $S, r, s$. If we would split up the integration region into subregions where the time differences have definite sign we would get rid of that complication at the price of considering many different contributions. This is exactly what happens in the diagrammatic approach and is the source of the proliferation of diagrams in high-order perturbation theory. 


\section{HOW TO MAKE THE BP APPROACH NUMERICALLY FEASIBLE}

Equation (30) together with Eqs. (20) and (39) specify how to calculate the $n$ th-order coefficient $e_{n}$ for the perturbative expansion of the polaron ground-state energy. Taken at face value one needs to evaluate a $3 n$-dimensional integral at large (asymptotic) values of the inverse temperature $\beta$. While this seems doable in principle, it is clear that in practice precise values of $e_{n}$ or the numerical feasibility of the whole approach need further improvements and refinements. As these practical questions have not been addressed at all in Bogoliubov and Plechko's paper [8] we will describe several steps crucial for success.

\section{A. Additional integrations}

It is obvious that any reduction in the dimensionality of the integral to be evaluated numerically will be of great help. As explained above the integrations over the times can only be performed by splitting the integration regions in many subregions leading to the time-honored diagrammatic approach. However, the dependence on the auxiliary variables $u_{i}$ is simple and analytic and therefore it is possible to perform some of the integrations over them by expanding the $n \times n$ determinant $\operatorname{det}_{n} A$ into cofactors [27]. For example, the dependence on $u_{n}$ is simply obtained by expanding with respect to the $n$th row (or column)

$$
\operatorname{det}_{n} A=u_{n} A_{n}+\operatorname{det}_{n} A\left(u_{n}=0\right),
$$

where $A_{n}$ denotes the determinant of the matrix which is obtained from $(A)$ by removing the $n$th row and the $n$th column, i.e., it is a special $(n-1) \times(n-1)$ determinant known as principal minor [28]. Therefore the integration over $u_{n}$ in Eq. (39) can be easily performed:

$$
\begin{aligned}
\mathcal{D}_{n}(1,2, \ldots, \underline{n}) & :=\int_{0}^{\infty} d u_{n} \operatorname{det}_{n}^{-3 / 2} A(1,2, \ldots, n) \\
& =\frac{2}{A_{n} \sqrt{\operatorname{det}_{n} A\left(u_{n}=0\right)}} .
\end{aligned}
$$

Here we use the short-hand notation $i:=\left(t_{i}, t_{i}^{\prime}, u_{i}\right)$ and the integration over $u_{n}$ is indicated by underlining the $n$th argument. The dependence on $u_{n-1}$ is obtained similarly:

$$
\begin{gathered}
A_{n}=u_{n-1} A_{n-1, n}+A_{n}\left(u_{n-1}=0\right), \\
\operatorname{det}_{n} A\left(u_{n}=0\right)=u_{n-1} A_{n-1}\left(u_{n}=0\right)+\operatorname{det}_{n} A\left(u_{n-1}=u_{n}=0\right) .
\end{gathered}
$$

Here $A_{n-1, n}$ denotes the determinant (principal minor) of the matrix which is obtained from $(A)$ by removing both the $(n$ $-1)$ th and the $n$th row and column. The subsequent integration over $u_{n-1}$ is therefore still an elementary one $\left(u_{n-1}=u_{n}\right.$ $=0$ is understood in all determinants from now on)

$$
\begin{aligned}
\mathcal{D}_{n} & (1,2, \ldots, \underline{n-1}, \underline{n}) \\
& :=\int_{0}^{\infty} d u_{n-1} \int_{0}^{\infty} d u_{n} \operatorname{det}_{n}^{-3 / 2} A(1,2, \ldots, n) \\
& =\int_{0}^{\infty} d u_{n-1} \frac{2}{u_{n-1} A_{n-1, n}+A_{n}} \frac{1}{\sqrt{u_{n-1} A_{n-1}+\operatorname{det}_{n} A}}
\end{aligned}
$$

but depends on the sign of the combination $A_{n} A_{n-1}$ $-A_{n-1, n} \operatorname{det}_{n} A$. This is fixed since all the coefficients in the integrand are principal minors of the positive semidefinite matrix $(A)$ which not only are non-negative themselves but also obey the Hadamard-Fischer inequality [Ref. [25], Eq. (7.8.9)]

$$
A_{n-1} A_{n} \geqslant A_{n-1, n} A
$$

$\left(A \equiv \operatorname{det}_{n} A\right)$. Therefore the integration over $u_{n-1}$ gives [see, e.g., Ref. [29], Eq. (192.11)]

$$
\mathcal{D}_{n}\left(1,2, \ldots, \underline{n-1, \underline{n})}=\frac{4}{\sqrt{A_{n-1, n} A_{n-1} A_{n}}} \frac{\arcsin \sqrt{x_{\mathrm{HF}}}}{\sqrt{x_{\mathrm{HF}}}},\right.
$$

where

$$
0 \leqslant x_{\mathrm{HF}}:=1-\frac{A_{n-1, n} A}{A_{n-1} A_{n}} \leqslant 1
$$

is non-negative and does not exceed unity as needed for a proper argument of the arcsin function.

Let us illustrate that for the case $n=2$ where all principal minors can be evaluated easily. With Eqs. (25) and (39) one then obtains

$$
\begin{aligned}
\lambda_{2}= & \frac{\alpha^{2}}{4 \pi} \int_{0}^{\beta} d t_{1} d t_{2} \int_{0}^{t_{1}} d t_{1}^{\prime} \int_{0}^{t_{2}} d t_{2}^{\prime} e^{-\left(t_{1}+t_{2}-t_{1}^{\prime}-t_{2}^{\prime}\right)} \\
& \times\left[\mathcal{D}_{2}(\underline{1}, \underline{2})-\mathcal{D}_{1}(\underline{1}) \mathcal{D}_{1}(\underline{2})\right] \\
= & \frac{\alpha^{2}}{\pi} \int_{0}^{\beta} d t_{1} d t_{2} \int_{0}^{t_{1}} d t_{1}^{\prime} \int_{0}^{t_{2}} d t_{2}^{\prime} e^{-\left(t_{1}+t_{2}-t_{1}^{\prime}-t_{2}^{\prime}\right)} \\
& \times \frac{1}{\sqrt{a_{11} a_{22}}} f_{2}\left(\frac{a_{12}}{\sqrt{a_{11} a_{22}}}\right),
\end{aligned}
$$

where

$$
f_{2}(x):=\frac{\arcsin (x)}{x}-1 .
$$

Thus the second cumulant (and therefore the second energy coefficient) would vanish without the nondiagonal matrix element $a_{12}$, i.e., the correlation between the times when the two phonons have been emitted (absorbed).

\section{B. Extrapolation for $\boldsymbol{\beta} \rightarrow \infty$}

A crucial question for the feasibility of the BP approach is how the asymptotic limit $\beta=\infty$ is reached. From Appendix B where the cases $n=1,2$ are treated explicitly we expect

$$
\lambda_{n}(\beta) \rightarrow \beta e_{n}+d_{n}+O\left(e^{-\beta / \sqrt{\beta}}\right)
$$


so that from Eq. (30) only a rather slow convergence to the asymptotic value is expected:

$$
e_{n}=\lim _{\beta \rightarrow \infty}\left[e_{n}+\frac{d_{n}}{\beta}\right] \text {. }
$$

This can be greatly improved not by dividing $\lambda_{n}(\beta)$ by $\beta$ but by taking the derivative of $\lambda_{n}(\beta)$, i.e., considering

$$
e_{n}(\beta):=\frac{(-)^{n+1}}{\alpha^{n} n !} \lim _{\beta \rightarrow \infty} \frac{\partial \lambda_{n}(\beta)}{\partial \beta}
$$

which approaches the asymptotic value exponentially

$$
e_{n}(\beta) \stackrel{\beta \rightarrow \infty}{\rightarrow} \frac{\partial}{\partial \beta}\left[\beta e_{n}+d_{n}+O\left(e^{-\beta / \sqrt{\beta}}\right)\right]=e_{n}+O\left(e^{-\beta / \sqrt{\beta}}\right)
$$

-at least in the analytical examples given in Appendix B for $n=1,2$.

We therefore will assume that for large enough $\beta$

$$
e_{n}(\beta) \rightarrow e_{n}+\frac{a_{n}}{\sqrt{\beta}} e^{-\beta}
$$

for all values of $n$ in the following. Alternatively, the behavior

$$
e_{n}(\beta) \stackrel{\beta \rightarrow \infty}{\rightarrow} e_{n}+\frac{a_{n}}{\beta^{\nu_{n}}} e^{-\beta}
$$

will be fitted to the numerical data if they are precise enough to determine also the power $\nu_{n}$.

Moreover, evaluating the differentiation with respect to $\beta$ also lowers the dimension of the integral which has to be evaluated numerically because the variable $\beta$ enters as upper limits of the multidimensional integral (47). Writing the corresponding cumulant as

$$
\begin{aligned}
\lambda_{n}= & (-)^{n} \frac{\alpha^{n}}{(4 \pi)^{n / 2}} \prod_{j=1}^{n}\left(\int_{0}^{\beta} d \sigma_{j} \int_{\sigma_{j} / 2}^{\beta-\sigma_{j} / 2} d \Sigma_{j}\right) \\
& \times F_{n}\left(\sigma_{1}, \Sigma_{1} ; \sigma_{2}, \Sigma_{2} ; \ldots ; \sigma_{n}, \Sigma_{n}\right)
\end{aligned}
$$

we find no contribution by differentiating the upper limit of the $\sigma_{j}$ integration since the range of $\Sigma_{j}$ then vanishes. Thus

$$
\begin{aligned}
\frac{\partial \lambda_{n}}{\partial \beta}= & \frac{(-\alpha)^{n}}{(4 \pi)^{n / 2}} \prod_{j=1}^{n}\left(\int_{0}^{\beta} d \sigma_{j}\right) \sum_{i}^{n} \prod_{k \neq i}\left(\int_{\sigma_{k} / 2}^{\beta-\sigma_{k} / 2} d \Sigma_{k}\right) \\
& \times\left|F_{n}\left(\sigma_{1}, \Sigma_{1} ; \sigma_{2}, \Sigma_{2} ; \ldots ; \sigma_{n}, \Sigma_{n}\right)\right|_{\Sigma_{i}=\beta-\sigma_{i} / 2} .
\end{aligned}
$$

For example, for $n=2$ we have

$$
\begin{aligned}
\frac{\partial \lambda_{2}}{\partial \beta}= & \frac{\alpha^{2}}{\pi} \int_{0}^{\beta} d \sigma_{1} d \sigma_{2} \frac{1}{\sqrt{\sigma_{1} \sigma_{2}}} e^{-\left(\sigma_{1}+\sigma_{2}\right)} \\
& \times\left[\int_{\sigma_{2} / 2}^{\beta-\sigma_{2} / 2} d \Sigma_{2} f_{2}\left(\frac{a_{12}}{\sqrt{\sigma_{1} \sigma_{2}}}\right)_{\Sigma_{1}=\beta-\sigma_{1} / 2}+(1 \leftrightarrow 2)\right] .
\end{aligned}
$$

\section{Symmetrization}

We may exchange simultaneously

$$
\sigma_{j}, \Sigma_{j} \leftrightarrow \sigma_{k}, \Sigma_{k}, \quad j \neq k=1, \ldots, n
$$

in the integrand of Eq. (67). There are $n$ ! ways of doing that and thus

$$
\begin{aligned}
\frac{\partial \lambda_{n}}{\partial \beta}= & \frac{(-\alpha)^{n}}{(4 \pi)^{n / 2}} \prod_{j=1}^{n}\left(\int_{0}^{\beta} d \sigma_{j}\right) \sum_{i}^{n} \prod_{k \neq i}\left(\int_{\sigma_{k} / 2}^{\beta-\sigma_{k} / 2} d \Sigma_{k}\right) \frac{1}{n !} \\
& \times\left.\underbrace{\sum_{\text {permut. }} F_{n}\left(\left\{\sigma_{j}, \Sigma_{k}\right\}\right)}_{=: F_{n}^{\text {symm }}\left(\left\{\sigma_{j}, \Sigma_{k}\right\}\right)}\right|_{\Sigma_{i}=\beta-\sigma_{i} / 2}
\end{aligned}
$$

and the domain of integration can be reduced [30]:

$$
\begin{aligned}
\frac{\partial \lambda_{n}}{\partial \beta}= & \frac{(-\alpha)^{n}}{(4 \pi)^{n / 2}} \int_{0}^{\beta} d \sigma_{1} \cdots \int_{0}^{\sigma_{n-1}} d \sigma_{n} \sum_{i}^{n} \prod_{k \neq i}\left(\int_{\sigma_{k} / 2}^{\beta-\sigma_{k} / 2} d \Sigma_{k}\right) \\
& \times\left. F_{n}^{\mathrm{symm}}\left(\left\{\sigma_{j}, \Sigma_{k}\right\}\right)\right|_{\Sigma_{i}=\beta-\sigma_{i} / 2} .
\end{aligned}
$$

Again taking $n=2$ as simple example we find from Eq. (68) that $F_{2}^{\text {symm }}=2 F_{2}$ as the integrand is already completely symmetric. Hence

$$
\begin{aligned}
\frac{\partial \lambda_{2}}{\partial \beta}= & \frac{2 \alpha^{2}}{\pi} \int_{0}^{\beta} d \sigma_{1} \int_{0}^{\sigma_{1}} d \sigma_{2} \frac{\exp \left(-\sigma_{1}-\sigma_{2}\right)}{\sqrt{\sigma_{1} \sigma_{2}}} \\
& \times\left[\left.\int_{\sigma_{1} / 2}^{\beta-\sigma_{1} / 2} d \Sigma_{1} f_{2}\left(\frac{a_{12}}{\sqrt{\sigma_{1} \sigma_{2}}}\right)\right|_{\Sigma_{1}=\beta-\sigma_{1} / 2}+(1 \leftrightarrow 2)\right],
\end{aligned}
$$

where we have used $a_{i i}=\sigma_{i}$. Further evaluation of Eq. (72) is presented in Appendix B. For $n>2$ we have to perform the symmetrization explicitly as the integrations over $u_{n}, u_{n-1}$ lead to a nonsymmetric integrand.

\section{Mapping}

Finally for Monte Carlo integration we need a mapping to bring all integration variables into the hypercube $[0,1]$. After some experimentation we have chosen

$$
u_{i}=\sigma_{i}\left(\frac{1}{\xi_{i}^{2}}-1\right), \quad i=1,2, \ldots,(n-2)
$$

and

$$
\sigma_{1}=\beta s_{1}^{2}, \quad \sigma_{i}=\sigma_{i-1} s_{i}^{2}, \quad i=2, \ldots, n,
$$

$$
\Sigma_{i}=\left(\beta-\sigma_{i}\right) S_{i}+\frac{1}{2} \sigma_{i}
$$

as transformation of the remaining variables. Here all $\xi_{i}, s_{i}, S_{i} \in[0,1]$. Equation (74) removes possible square-root singularities which are seen in the examples for $n=1,2$ in Appendix B-these are integrable analytically but would pose severe problems for numerical integration. More refined 
TABLE I. Third-order energy coefficient $e_{3}(\beta)$ from the derivative of the third cumulant as a function of the inverse temperature $\beta$. The numerical results were obtained with the Monte Carlo routine VEGAS for evaluating the full six-dimensional integral. Numbers in parenthesis are the estimated errors in units of the last digit. The last column gives the $\chi^{2}$ per degree of freedom $\left(N_{\mathrm{DF}}\right)$ monitored during the iterations. This should be close to one if the iterations are consistent with each other.

\begin{tabular}{lcc}
\hline \hline$\beta$ & $-e_{3}(\beta) \times 10^{3}$ & $\chi^{2} / N_{\mathrm{DF}}$ \\
\hline 4.0 & $0.7474(5)$ & 0.969 \\
4.5 & $0.7704(7)$ & 0.876 \\
5.0 & $0.7846(8)$ & 0.836 \\
5.5 & $0.7934(10)$ & 0.837 \\
6.0 & $0.7987(11)$ & 0.821 \\
6.5 & $0.8017(13)$ & 0.792 \\
7.0 & $0.8033(15)$ & 0.768 \\
7.5 & $0.8039(17)$ & 0.775 \\
8.0 & $0.8041(19)$ & 0.772 \\
\hline \hline
\end{tabular}

mappings of the relative times (for example, to include the exponential suppression) have been tried but did not result in significant improvements.

\section{NUMERICAL RESULTS}

\section{A. A test: $e_{3}$}

We have tested our approach by determining the thirdorder coefficient $e_{3}$ which has been calculated by Smondyrev [16] with later improvements in accuracy [17]. Table I lists the values of $e_{3}(\beta)$ obtained by Monte Carlo integration using the classic VEGAS program [31] with $n_{\mathrm{MC}}=4.9 \times 10^{8}$ function calls per iterations. We have used 100 iterations for each $\beta$ value. Thus the total number of function calls was

$$
n_{\mathrm{tot}}^{(3)}=n_{\mathrm{MC}} n_{\mathrm{it}}=4.9 \times 10^{10} \text {. }
$$

Figure 2 shows that $e_{3}(\beta)$ monotonically approaches Smondyrev's value with increasing $\beta$. The sheer fact that $e_{3}(\beta)$ converges to a constant value at large $\beta$ is a good signal: individual moments $m_{n}$ would behave as $\beta^{n}$ for large values of $\beta$ but the construction of the cumulants takes away all these $\beta$ powers except the linear one which contains the information about the ground state energy.

We have fitted these data with the ansatz (64) which, of course, only holds for asymptotic values of $\beta$. Therefore the lower limit $\beta_{\min }$ of the fit range $\left[\beta_{\min }, \beta_{\max }\right]$ was successively raised until the $\chi^{2} / N_{\mathrm{DF}}$ of the fit reached a minimum. This is displayed in Table II. If $\beta_{\min }$ is too close to $\beta_{\max }$ the degrees of freedom decrease which should cause the $\chi^{2} / N_{\mathrm{DF}}$ to increase in turn [32]. This fitting strategy yielded

$$
e_{3}=-0.8056(8) \times 10^{-3} \text {. }
$$

If we allow the more general ansatz (65) we obtain as best fit

$$
e_{3}=-0.8055(6) \times 10^{-3}
$$

and

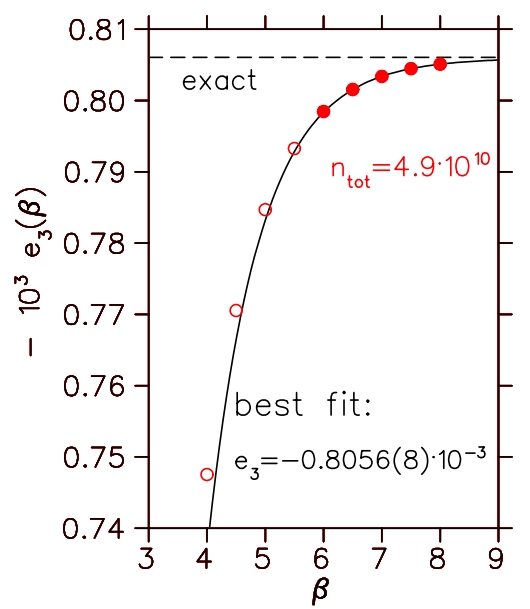

FIG. 2. (Color online) Monte Carlo results for the derivative of the third cumulant as a function of the Euclidean time (inverse temperature) $\beta$. The total number of function calls is denoted by $n_{\text {tot }}$ and the full (open) circles are the points used (not used) in the fit (see Table II).

$$
\nu_{3}=0.55(3) \text {. }
$$

The above error estimates may be a little bit optimistic since we have taken the VEGAS errors at face value. In addition, the power $\nu_{3}$ and the parameter $a_{3}$ in the fit function (65) turn out to be highly correlated. Nevertheless the $\exp (-\beta) / \sqrt{\beta}$ behavior also seems to hold for higher cumulants and the extrapolated result is in good agreement with Smondyrev's analytical result (8). The main message of this test therefore is that (our implementation of) the BP method is working and able to give accurate values for the perturbative expansion of the ground-state energy of a polaron.

\section{B. A new coefficient: $e_{4}$}

When applying the previous approach to the calculation of the first unknown coefficient $e_{4}$ an unpleasant outcome is found: as seen in Fig. 3 for a fixed value of $\beta=5$ the convergence with the number of function calls is very slow. Since the cancellations in the integrand are more severe for the large $\beta$ which is needed for determining $e_{4}$ only a very rough determination of this coefficient was possible in acceptable CPU time.

Fortunately a solution was found by performing the remaining integrations over $u_{i}, i=1,2$ by a deterministic inte-

TABLE II. Extrapolation of $e_{3}(\beta)$ to $\beta=\infty$ using the data from Table I, the fitting range $\beta \in\left[\beta_{\min }, \beta_{\max }\right]$ and the fixed power $\nu_{3}$ $=0.5$ in the ansatz (64). The last column gives the $\chi^{2} / N_{\mathrm{DF}}$ of the two-parameter fit where $N_{\mathrm{DF}}=$ number of data points -2 .

\begin{tabular}{lccc}
\hline \hline$\beta_{\min }$ & $\beta_{\max }$ & $-e_{3} \times 10^{3}$ & $\chi^{2} / N_{\mathrm{DF}}$ \\
\hline 4.0 & 8.0 & $0.8043(6)$ & 0.989 \\
4.5 & 8.0 & $0.8052(7)$ & 0.138 \\
5.0 & 8.0 & $0.8056(8)$ & 0.048 \\
5.5 & 8.0 & $0.8055(10)$ & 0.058 \\
\hline
\end{tabular}




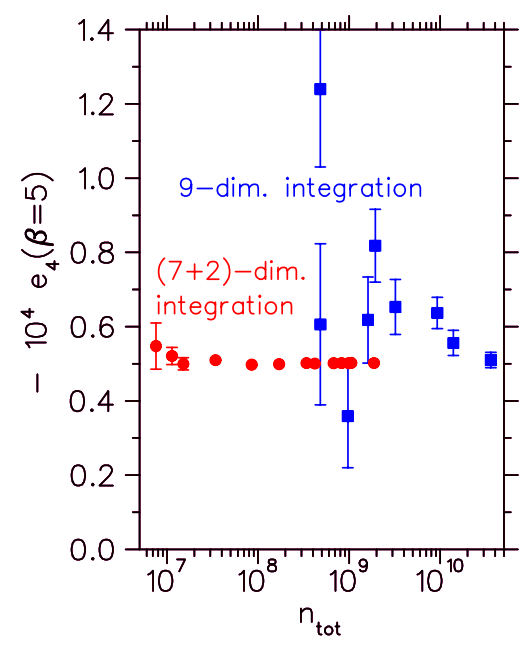

FIG. 3. (Color online) Convergence of the fourth-order coefficient $e_{4}(\beta=5)$ for a fixed value of the inverse temperature $\beta$ as function of the total number of function calls $n_{\text {tot }}$. Square (blue) points denote the case where the full nine-dimensional integral was evaluated by the Monte Carlo routine VEGAS, (red) circles show the result if two of the integrations are done by the deterministic tanhsinh-quadrature rule and the rest stochastically.

gration routine. While such an option is not available for the time integrations for which the integrand is nondifferentiable (see Fig. 1) it is possible for the integration over the auxiliary variables $u_{i}$ where the dependence is an analytic one [see Eqs. (39) and (40)].

We have used the powerful tanh-sinh integration procedure [33] which-after a judicious transformation of variables - is nothing else than the trapezoidal approximation to the transformed integral

$$
\int_{a}^{b} d x f(x) \approx h \frac{b-a}{2} \sum_{k=-k_{\max }}^{k_{\max }} w_{k} f\left(\frac{b+a}{2}+\frac{b-a}{2} x_{k}\right)
$$

with precalculated abscissae $x_{k}$ and weights $w_{k}$. Since this quadrature rule seems not to be very well known (see, however, Ref. [34]) Appendix C gives a short account of its basic features together with details of our implementation. Having in mind an application to our multidimensional case the convergence rate with the number of function calls

$$
n_{t}=2 k_{\max }+1
$$

is of paramount interest. In the one-dimensional case the error may decrease as fast as $\exp \left(-c n_{t} / \ln n_{t}\right)[35,36]$ depending on the analyticity domain of the transformed function $f(x)$. However, without any knowledge about that and in a multidimensional application, such an error estimate is of no help and we have to test the convergence of the quadrature rule with increasing $n_{t}$. The outcome is also shown in Fig. 3 as function of

$$
n_{\mathrm{tot}}^{(4)}=n_{t}^{2} n_{\mathrm{MC}} n_{\mathrm{it}}
$$

and demonstrates an improvement by two orders of magnitude compared to the previous approach which fully evaluated the nine-dimensional integral by stochastic methods.

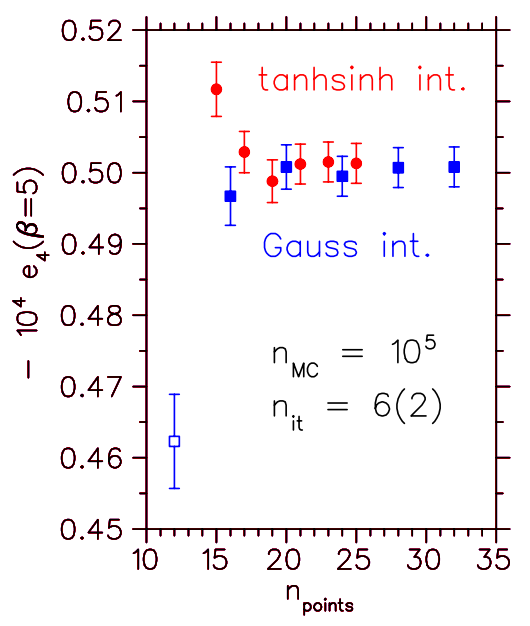

FIG. 4. (Color online) Comparison of deterministic integration routines for $e_{4}(\beta=5)$ as a function of the number of integration points. The number of Monte Carlo calls $\left(n_{\mathrm{MC}}\right)$ and iterations $\left(n_{\mathrm{it}}\right)$ is kept fixed. An open symbol indicates a Monte Carlo result with inconsistent iterations.

Figure 4 shows a comparison with Gaussian integration which also gives fairly good results.

This improvement now allows a much more precise determination of the coefficient $e_{4}$ (and, of course, also of the third-order coefficient [37]). Table III contains the data for $e_{4}(\beta)$ from $\beta=4$ to $\beta=8$ each with 12 iterations; the first 2 iterations were used for establishing the optimal grid while the following 10 were utilized for the statistics [denoted by $n_{\mathrm{it}}=12(2)$ in the following]. In addition to the classic VEGAS program (as in the previous test for $e_{3}$ ) we also have used the VEGAS program from the CUBA library [38] which employs Sobol quasirandom numbers. This allowed to extend the range of inverse temperatures up to $\beta=10$. Typical run times were about 1 day on a $2.4 \mathrm{GHz}$ PC. It is seen that for all $\beta$ there is agreement between the two data sets within the error bars. Despite larger statistics and higher accuracy in the deterministic integration the VEGAS (CUBA) routine returns larger errors which reflects our experience that the VEGAS (classic) error estimate often is too optimistic. This is also corroborated by the observation that at various $\beta$ values the VEGAS (classic) results have an unacceptable large $\chi^{2} / N_{\mathrm{DF}}$ indicating inconsistencies between different iterations within the given error bars.

But also for the VEGAS (CUBA) results the probability that the error is unreliable increases with the value of $\beta$. This just reflects the fact that the cancellations inside the integrand are becoming more and more challenging at high $\beta$. Fitting the VEGAS (CUBA) data with the asymptotic ansatz (64) yields

$$
e_{4}=-0.5328(9) \times 10^{-4} \text {. }
$$

Data and best fit are shown in Fig. 5. The more general ansatz (65) leads to

$$
e_{4}=-0.5330(7) \times 10^{-4}
$$

with $\nu_{4}=0.35(7)$. We therefore take 
TABLE III. Same as in Table I but for the fourth-order term $e_{4}(\beta)$. The numerical results were obtained by a combination of deterministic and stochastic integration of the nine-dimensional integral (see text). Two different versions of the VEGAS program have been used: the classic one with pseudorandom numbers and the CUBA version with Sobol quasirandom numbers. The number of points in the deterministic tanh-sinh integration is denoted by $n_{t}$. In the VEGAS (classic) evaluation $n_{\mathrm{it}}=12(2)$ iterations were used at each $\beta$ value. Data marked by an asterisk have an unacceptable $\chi^{2} / N_{\mathrm{DF}}$ (underlined) indicating that the iterations do not lead to a consistent error estimate. The last column gives the probability $p$ that the error estimate for the VEGAS (CUBA) results is not reliable $(p<0.95$ is considered to be safe).

\begin{tabular}{lcccc}
\hline \hline & \multicolumn{2}{c}{$\begin{array}{c}\text { VEGAS (classic): } \\
n_{\mathrm{MC}}=4.7 \times 10^{5} \\
n_{t}=23\end{array}$} & \multicolumn{2}{c}{$\begin{array}{c}\text { VEGAS (Cuba): } \\
n_{\mathrm{MC}}=3 \times 10^{6} \\
n_{t}=25\end{array}$} \\
\cline { 2 - 5 }$\beta$ & $-e_{4}(\beta) \times 10^{4}$ & $\chi^{2} / N_{\mathrm{DF}}$ & $-e_{4}(\beta) \times 10^{4}$ & $p$ \\
\hline 4.0 & $0.4549(6)$ & 0.637 & $0.4563(10)$ & 0.164 \\
4.5 & $0.4828(7)$ & 0.995 & $0.4839(11)$ & 0.157 \\
5.0 & $0.5013(8)^{*}$ & $\underline{1.404}$ & $0.5020(12)$ & 0.170 \\
5.5 & $0.5129(8)$ & 1.087 & $0.5136(13)$ & 0.406 \\
6.0 & $0.5193(9)^{*}$ & $\underline{1.739}$ & $0.5209(14)$ & 0.413 \\
6.5 & $0.5239(9)^{*}$ & $\underline{1.488}$ & $0.5254(15)$ & 0.480 \\
7.0 & $0.5271(10)$ & 0.977 & $0.5287(16)$ & 0.534 \\
7.5 & $0.5293(10)^{*}$ & $\underline{1.830}$ & $0.5304(18)$ & 0.588 \\
8.0 & $0.5309(11)^{*}$ & $\underline{1.520}$ & $0.5309(17)$ & 0.646 \\
8.5 & & & $0.5313(19)$ & 0.387 \\
9.0 & & & $0.5320(19)$ & 0.355 \\
9.5 & & & $0.5327(20)$ & 0.483 \\
10.0 & & & $0.5333(19)$ & 0.553 \\
\hline
\end{tabular}

$$
e_{4}=-0.533(1) \times 10^{-4}
$$

as our final result.

\section{A further step: $e_{5}$}

We have extended the BP approach to the calculation of the fifth-order coefficient $e_{5}(\beta)$. Numerically this is much more challenging than the fourth-order calculation since these coefficients drop by roughly one order of magnitude in each order. This has to be achieved by cancellation in a $12-$ dimensional integral over a much more complicated integrand leading to much larger CPU times. Nevertheless the combination of deterministic integration and Monte Carlo integration leads to reasonable results. Figure 6 shows a slight advantage of the tanh-sinh integration rule compared to Gaussian integration. Of course, due to the more severe cancellations in the 12-dimensional integrand higher accuracy, i.e., a larger number of deterministic integration points is needed. At the same time the number of Monte Carlo calls cannot be as large as before to avoid excessive running times.

Another numerical problem which already plagued the numerics for $n=4$ (and to a much lesser extent $n=3$ ) became more severe in the present case: due to round-off errors the

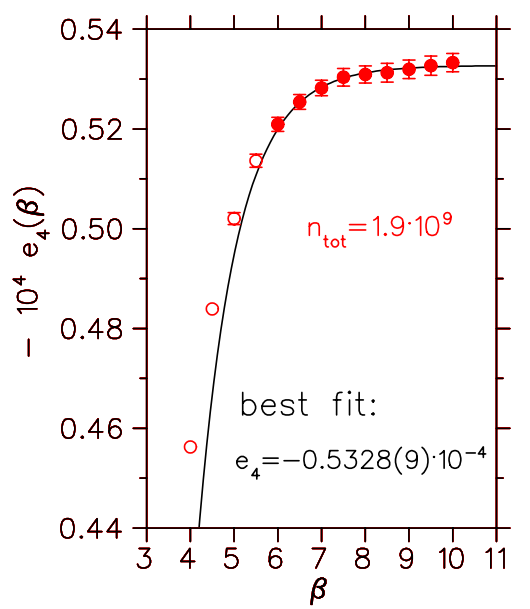

FIG. 5. (Color online) Same as in Fig. 2 but for the derivative of the fourth cumulant. The plotted data points are the VEGAS (CUBA) results from Table III.

Hadamard-Fisher inequality (55) was not fulfilled exactly all the time: negative values down to

$$
x_{\mathrm{HF}}^{\min }=-3.1 \times 10^{-9}
$$

were recorded in double-precision arithmetic. Fortunately, this "digit-deficiency error" (see Appendix B of Ref. [39]) does not affect the outcome of the Monte Carlo runs: checks have shown that $e_{5}(\beta=4)$ comes out the same whether the negative argument is set to zero or the absolute value of $x_{\mathrm{HF}}$ is taken. In addition, the use of quadruple precision gives a consistent result (within error bars) but reduces the violation of the Hadamard-Fisher inequality considerably-at the price of a 20-fold longer running time.

The data are collected in Table IV and show that at high $\beta$ it becomes more and more difficult to get consistent numerical results. Typical run times for each $\beta$ value were about 1 month on a $3.0 \mathrm{GHz}$ xeon machine. With the Intel ifort compiler some loops could be vectorized leading to a reduc-

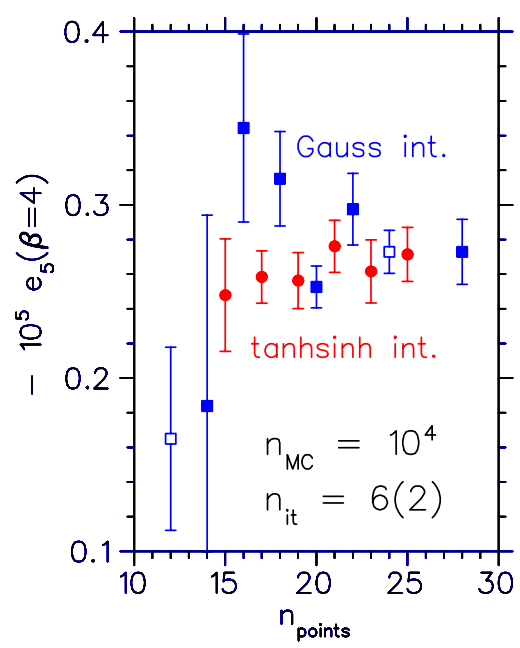

FIG. 6. (Color online) Comparison of tanh-sinh and Gaussian integration for the derivative of the fifth cumulant at $\beta=4$. Notation as in Fig. 4. 
TABLE IV. Same as in Table III but for the fifth-order term $e_{5}(\beta)$. For all deterministic numerical integrations $n_{t}=25$ integration points were used in the tanh-sinh integration routine. The Monte Carlo integrations were either done with the VEGAS (CUBA) program $\left(n_{\mathrm{MC}}=1.5 \times 10^{5}\right)$ or the classic VEGAS routine with $n_{\mathrm{MC}}=7.9 \times 10^{4}$, $n_{\mathrm{it}}=6(2)$ except for the data in boldface for which $n_{\mathrm{MC}}=9.8 \times 10^{4}$, $n_{\mathrm{it}}=5(2)$.

\begin{tabular}{lcccc}
\hline \hline & VEGAS (classic) & & VEGAS (CUBA) \\
$\beta$ & $-e_{5}(\beta) \times 10^{5}$ & $\chi^{2} / N_{\mathrm{DF}}$ & $-e_{5}(\beta) \times 10^{5}$ & $p$ \\
\hline 4.0 & $0.290(4)$ & 0.240 & $0.295(10)$ & 0.369 \\
4.5 & $0.337(7)$ & 1.052 & $0.317(25)$ & 0.722 \\
5.0 & $0.347(6)$ & 0.537 & $0.349(18)$ & 0.353 \\
5.5 & $0.365(14)$ & 0.177 & $0.330(22)$ & 0.365 \\
6.0 & $0.367(7)$ & 0.287 & $0.327(26)$ & 0.657 \\
6.5 & $0.361(8)$ & 0.846 & $0.370(18)^{*}$ & $\underline{0.956}$ \\
7.0 & $0.365(10)$ & 0.984 & $0.394(30)$ & 0.404 \\
7.5 & $0.390(13)$ & 1.296 & $0.390(42)$ & 0.329 \\
& $\mathbf{0 . 3 9 0 ( 9 )}$ & $\mathbf{0 . 5 9 2}$ & & \\
8.0 & $0.366(10) *$ & $\underline{2.514}$ & $0.367(35)$ & 0.326 \\
& $\mathbf{0 . 3 8 0 ( 1 5 ) *}$ & $\underline{\mathbf{1 . 7 5 5}}$ & & \\
\hline \hline
\end{tabular}

tion in CPU time by more than a factor of 2 . If we exclude the data with $\chi^{2} / N_{\mathrm{DF}}>1.3$ and $p>0.9$ we obtain from a fit with $\nu_{5}=0.5$ fixed

$$
e_{5}=-0.378(4) \times 10^{-5} \text {. }
$$

This is shown in Fig. 7 together with the corresponding values of

$$
n_{\mathrm{tot}}^{(5)}=n_{t}^{3} n_{\mathrm{MC}} n_{\mathrm{it}}
$$

for the different data from Table IV. It is not possible to determine the exponent $\nu_{5}$ unambiguously from the data which scatter too much. Taking a range of reasonable values for $\nu_{5}$ we end up with

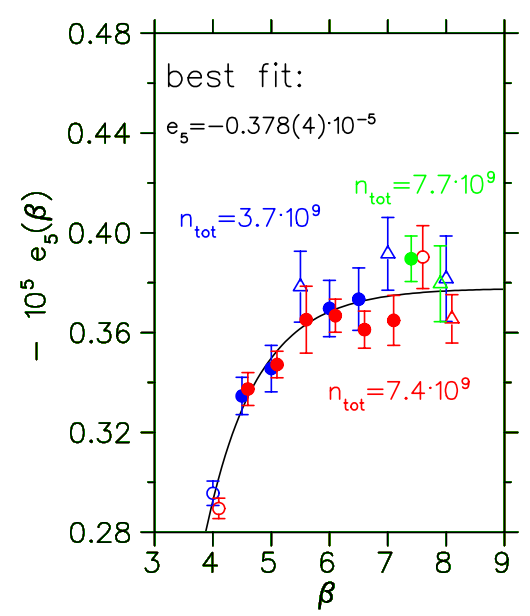

FIG. 7. (Color online) Same as in Fig. 2 but for the derivative of the fifth cumulant. Data points with open triangles are from statistically inconsistent Monte Carlo iterations (see Table IV) and are not used in the fit.

$$
e_{5}=-0.38(2) \times 10^{-5}
$$

as final result for the fifth-order energy coefficient. It is obvious that the given error is more an educated (and conservative) guess than a precise outcome of the fit.

\section{CONCLUSION AND OUTLOOK}

We have shown that the Bogoliubov-Plechkov (BP) approach to calculate perturbative coefficients without diagrams works for the polaron problem (a simple field theory of electrons and phonons) if it is combined with several simple but crucial "tricks" to enhance the numerical feasibility and convergence. There is no indication that higher cumulants are "unbounded from below" as was reported in Ref. [40] in a much simpler anharmonic oscillator model [41]. It is worthwhile to point out the advantages and disadvantages of the BP approach compared to the standard perturbative method.

While in the diagrammatic approach a factorial increasing number of individual (zero-temperature) diagrams adds up to the final result, much fewer terms (moments) [see, e.g., Eqs. (27) and (28)] must cancel inside the finite-temperature integral in the BP approach to obtain a result which is linear in $\beta$ so that the perturbative ground-state energy of the polaron can be determined. Of course, diagrams can be calculated exactly at zero temperature whereas in the BP approach the extrapolation $\beta \rightarrow \infty$ must be performed numerically. We have demonstrated that by evaluating the derivative of the various cumulants, an exponential convergence to the zerotemperature limit can be exploited. Two new perturbative coefficients $e_{4}$ and $e_{5}$ for the ground-state energy of a polaron have been obtained in this way and compared to results from Feynman's approximate treatment.

It should be emphasized that the BP approach says nothing about the convergence of the perturbative series as it works in a fixed order. For the polaron case it is known that the ground-state energy is an analytic function of the coupling constant [42] but this is not necessary and systems where the perturbative expansion is known (or suspected) not to converge could be treated as well. Indeed, there is some hope that the methods which in the present work have been applied successfully for a simple nonrelativistic field theory may also be suited for relativistic field theories such as QED and QCD if these are formulated in the worldline formalism. Renormalization of the occurring divergencies is the main new challenge which is under investigation.

\section{ACKNOWLEDGMENTS}

Many thanks to Michael Spira who supplied his version of the classic VEGAS program and to Valery Markushin for help with compiler optimization which led to a considerable speed up of the calculations. I am also indebted to Dr. Plechko who informed me about his previous work in Ref. [8] and made some valuable remarks.

\section{APPENDIX A: ENERGY COEFFICIENTS FROM A QUADRATIC TRIAL ACTION}

Here we briefly describe the results obtained with Feynman's variational method and with the best quadratic ap- 
proximation [43]. Employing Jensen's inequality and working out the various path integral averages one finds that the true ground-state energy is below the variational energy

$$
E_{0} \leqslant E_{t}=\Omega+V
$$

where

$$
\begin{gathered}
\Omega=\frac{3}{2 \pi} \int_{0}^{\infty} d E\left[\ln A(E)+\frac{1}{A(E)}-1\right], \\
V=-\frac{\alpha}{\sqrt{\pi}} \int_{0}^{\infty} d \sigma \frac{\exp (-\sigma)}{\left[\mu^{2}(\sigma)\right]^{1 / 2}} .
\end{gathered}
$$

Here $A(E)$ is the "profile function" which is related to the retardation function by

$$
A(E)=1+8 \int_{0}^{\infty} d \sigma f(\sigma) \frac{\sin ^{2}(E \sigma / 2)}{E^{2}}
$$

and $\mu^{2}(\sigma)$ the "pseudotime" [44] given by

$$
\mu^{2}(\sigma)=\frac{4}{\pi} \int_{0}^{\infty} d E \frac{1}{A(E)} \frac{\sin ^{2}(E \sigma / 2)}{E^{2}} .
$$

In Feynman's original work the retardation function is parametrized as

$$
f_{F}(\sigma)=C e^{-w \sigma}
$$

which has the advantage that profile function, pseudotime and the kinetic term can be calculated analytically:

$$
\begin{gathered}
A_{F}(E)=\frac{v^{2}+E^{2}}{w^{2}+E^{2}}, \quad \mu_{F}^{2}(\sigma)=\frac{w^{2}}{v^{2}} \sigma+\frac{v^{2}-w^{2}}{v^{3}}\left(1-e^{-v \sigma}\right), \\
\Omega_{F}=\frac{3}{4} \frac{(v-w)^{2}}{v} .
\end{gathered}
$$

Here $v=\sqrt{w^{2}+4 C / w}$ is used as parameter instead of the original strength $C$. Setting $\sigma=s^{2}$ we thus have to minimize

$$
\begin{aligned}
E_{F}(v, w)= & \frac{3}{4} \frac{(v-w)^{2}}{v}-\frac{2 \alpha}{\sqrt{\pi} w} \frac{v}{0} \int_{0}^{\infty} d s e^{-s^{2}} \\
& \times\left[1+\frac{v^{2}-w^{2}}{v w^{2}} \frac{1-e^{-v s^{2}}}{s^{2}}\right]^{-1 / 2} \\
= & \frac{3}{4} \frac{(v-w)^{2}}{v}-\alpha \frac{v}{w} \sum_{n=0}^{\infty} b_{n}(v)\left(\frac{v^{2}-w^{2}}{v w^{2}}\right)^{n},
\end{aligned}
$$

where [45]

$$
\begin{aligned}
b_{n}(v) & =\frac{2}{\sqrt{\pi}}\left(\begin{array}{c}
-1 / 2 \\
n
\end{array}\right) \int_{0}^{\infty} d s e^{-s^{2}}\left(\frac{1-e^{-v s^{2}}}{s^{2}}\right)^{n} \\
& =\frac{1}{n !} \sum_{k=0}^{n}\left(\begin{array}{l}
n \\
k
\end{array}\right)(-)^{k} \sqrt{1+k v^{2 k-1}} .
\end{aligned}
$$

For the actual calculation it is more convenient to introduce $c=v^{2} / w^{2}-1=4 C / w^{3}$ so that

$$
E_{F}(c, v)=\frac{3}{4} v\left(1-\frac{1}{\sqrt{1+c}}\right)^{2}-\alpha \sqrt{1+c} \sum_{n=0}^{\infty} b_{n}(v)\left(\frac{c}{v}\right)^{n}
$$

and to expand the parameters as

$$
\begin{gathered}
c=c_{1} \alpha+c_{2} \alpha^{2}+\cdots, \\
v=v_{0}+v_{1} \alpha+v_{2} \alpha^{2}+\cdots .
\end{gathered}
$$

Including terms up to second order in $\alpha$ one finds $v_{0}=3, c_{1}$ $=4 / 27$, and $E_{F} \rightarrow-\alpha-\alpha^{2} / 81$. In higher orders the minimization always leads to linear equations for the coefficients $c_{n}, v_{n}$ so that they can be solved easily. With the help of a symbolic algebra system (such as MAPLE) the higher-order coefficients $e_{n}^{F}$ can then be evaluated in a straightforward manner and are given in Eqs. (14) and (15).

It should be noted that in lowest order also the retardation parameter $w=3+O(\alpha)$ instead of $w \rightarrow 1$ as one would have expected naively. This is due to the wrong small- $\sigma$ behavior in the ansatz (A5) for Feynman's retardation function and would be corrected by an "improved parametrization" [10]

$$
f_{I}(\sigma)=x_{I} \frac{\alpha}{6 \sqrt{\pi}} \frac{\exp \left(-w_{I} \sigma\right)}{\sigma^{3 / 2}} .
$$

It is easy to check that both $x_{I}, w_{I} \rightarrow 1+O(\alpha)$ for small $\alpha$. However, one can do even better by letting the functional form of the retardation function free. In this "best quadratic approximation" [43] one finds

$$
f_{\text {best }}(\sigma)=\frac{\alpha}{6 \sqrt{\pi}} \frac{\exp (-\sigma)}{\left[\mu_{\text {best }}^{2}(\sigma)\right]^{3 / 2}}
$$

for which Eq. (A12) is a convenient approximation since one knows that generally

$$
\mu^{2}(\sigma) \stackrel{\sigma \rightarrow 0}{\longrightarrow} U_{0}(\sigma) \equiv \sigma .
$$

Indeed, inserting $U_{0}(\sigma)$ into the virial expression for the polaron ground state energy [43]

$$
E_{\text {virial }}=-\frac{\alpha}{\sqrt{\pi}} \int_{0}^{\infty} d \sigma \frac{\exp (-\sigma)}{\sqrt{\mu^{2}(\sigma)}}\left(\frac{3}{2}-\sigma\right)
$$

one obtains $E_{\text {virial }} \rightarrow-\alpha$ for $\alpha \rightarrow 0$, i.e., $e_{1}^{\text {best }}=-1$.

In second order we need the first-order change of the profile function and pseudotime

$$
A_{\text {best }}(E)=1+\alpha a_{1}(E)+\alpha^{2} a_{2}(E)+\cdots,
$$

$$
\mu_{\text {best }}^{2}(\sigma)=\sigma+\alpha U_{1}(\sigma)+\alpha^{2} U_{2}(\sigma)+\cdots .
$$

From the connection (A3) between profile function and retardation function one finds

$$
a_{1}(E)=\frac{4}{3 \sqrt{\pi}} \int_{0}^{\infty} d \sigma \frac{\exp (-\sigma)}{\sigma^{3 / 2}} \frac{\sin ^{2} E \sigma / 2}{E^{2}}
$$

and therefore from Eq. (A4) 


$$
\begin{aligned}
U_{1}(\sigma)= & -\frac{4}{3 \sqrt{\pi}} \frac{4}{\pi} \int_{0}^{\infty} d \sigma^{\prime} \frac{\exp \left(-\sigma^{\prime}\right)}{\sigma^{\prime 3 / 2}} \\
& \times \int_{0}^{\infty} d E \frac{\sin ^{2} E \sigma / 2 \sin ^{2} E \sigma^{\prime} / 2}{E^{4}} \\
= & -\frac{1}{9 \sqrt{\pi}} \int_{0}^{\infty} d \sigma^{\prime} \frac{\exp \left(-\sigma^{\prime}\right)}{\sigma^{\prime 3 / 2}} \sigma_{<}^{2}\left(3 \sigma_{>}-\sigma_{<}\right),
\end{aligned}
$$

where $\sigma_{<}=\min \left(\sigma, \sigma^{\prime}\right)$. It is possible to express the last integral exactly in terms of error functions and exponentials. However, for the calculation of the second-order energy it is better to plug this expression directly into the virial energy (A15) and expand $\left[\mu_{\text {best }}^{2}(\sigma)\right]^{-1 / 2}$ up to first order.

Substituting $\sigma=s^{2}, \sigma^{\prime}=s^{\prime 2}$ we then obtain

$$
\begin{aligned}
E_{0}^{\mathrm{best}}= & -\alpha-\frac{2 \alpha^{2}}{9 \pi} \int_{0}^{\infty} d s \frac{3 / 2-s^{2}}{s^{2}} e^{-s^{2}} \\
& \times \int_{0}^{\infty} d s^{\prime} \frac{s_{<}^{4}\left(3 s_{>}^{2}-s_{<}^{2}\right)}{s^{\prime 2}} e^{-s^{\prime 2}}+O\left(\alpha^{3}\right),
\end{aligned}
$$

where $s_{<}=\min \left(s, s^{\prime}\right)$. Introducing polar coordinates $s$ $=r \cos \phi, s^{\prime}=r \sin \phi$ the integral with $\pi / 4 \leqslant \phi \leqslant \pi / 2$ can be combined with the one in which $0 \leqslant \phi \leqslant \pi / 4$ and one obtains

$$
\begin{aligned}
e_{2}^{\text {best }}= & -\frac{2}{9 \pi} \int_{0}^{\infty} d r r^{3}\left(3-r^{2}\right) e^{-r^{2}} \\
& \times \int_{0}^{\pi / 4} d \phi \tan ^{2} \phi\left(3 \cos ^{2} \phi-\sin ^{2} \phi\right) \\
= & -\left(\frac{1}{12}-\frac{2}{9 \pi}\right) .
\end{aligned}
$$

Higher-order terms may be calculated numerically by using a delay-type equation for the pseudotime which was found in the variational approximation for worldline QED and dubbed "variational Abraham-Lorentz equation" (VALE) [46]. It can be easily checked that the corresponding equation for the three-dimensional polaron case is

$$
\begin{aligned}
\ddot{\mu}_{\text {best }}^{2}(\sigma) & \equiv \frac{d^{2} \mu^{2}(\sigma)}{d \sigma^{2}}=\frac{4}{3} \int_{0}^{\infty} d \sigma^{\prime} \frac{\delta V}{\delta \mu^{2}\left(\sigma^{\prime}\right)} X\left(\sigma, \sigma^{\prime}\right) \\
& =\frac{2 \alpha}{3 \sqrt{\pi}} \int_{0}^{\infty} d \sigma^{\prime} \frac{\exp \left(-\sigma^{\prime}\right)}{\left[\mu_{\text {best }}^{2}\left(\sigma^{\prime}\right)\right]^{3 / 2}} X\left(\sigma, \sigma^{\prime}\right),
\end{aligned}
$$

where

$$
X\left(\sigma, \sigma^{\prime}\right):=\mu_{\text {best }}^{2}(\sigma)-\frac{1}{2} \mu_{\text {best }}^{2}\left(\sigma+\sigma^{\prime}\right)-\frac{1}{2} \mu_{\text {best }}^{2}\left(\left|\sigma-\sigma^{\prime}\right|\right)
$$

is the delayed pseudotime (due to the phonon degrees of freedom which have been integrated out). Equation (A22) may be integrated with the boundary conditions $\mu^{2}(0)$ $=0, \dot{\mu}^{2}(0)=1$ to give

$$
\begin{aligned}
\mu_{\text {best }}^{2}(\sigma)= & \sigma+\frac{2 \alpha}{3 \sqrt{\pi}} \int_{0}^{\sigma} d \sigma^{\prime}\left(\sigma-\sigma^{\prime}\right) \\
& \times \int_{0}^{\infty} d \sigma^{\prime \prime} \frac{\exp \left(-\sigma^{\prime \prime}\right)}{\left[\mu_{\text {best }}^{2}\left(\sigma^{\prime \prime}\right)\right]^{3 / 2}} X\left(\sigma^{\prime}, \sigma^{\prime \prime}\right) .
\end{aligned}
$$

This gives an iterative scheme to calculate the perturbative terms (A17) for the pseudotime and eliminates the corresponding expansion (A16) for the profile function completely. Expanding in powers of $\alpha$ we obtain

$$
\begin{aligned}
U_{n}(\sigma)= & \frac{2}{3 \sqrt{\pi}} \int_{0}^{\sigma} d \sigma^{\prime}\left(\sigma-\sigma^{\prime}\right) \\
& \times \int_{0}^{\infty} d \sigma^{\prime \prime \prime} \frac{\exp \left(-\sigma^{\prime \prime}\right)}{\sigma^{\prime \prime 3 / 2}} Y_{n}\left(\sigma^{\prime}, \sigma^{\prime}\right), \quad n \geqslant 1 .
\end{aligned}
$$

Defining the delayed pseudotime of order $n$ as

$$
\begin{gathered}
X_{n}\left(\sigma^{\prime}, \sigma^{\prime \prime}\right):=U_{n}\left(\sigma^{\prime}\right)-\frac{1}{2} U_{n}\left(\sigma^{\prime}+\sigma^{\prime \prime}\right)-\frac{1}{2} U_{n}\left(\left|\sigma^{\prime}-\sigma^{\prime \prime}\right|\right), \\
n=0,1, \ldots,
\end{gathered}
$$

the functions $Y_{n}$ are given by (for simplicity all arguments are suppressed)

$$
\begin{gathered}
Y_{1}=X_{0}, \quad Y_{2}=X_{1}-\frac{3}{2} \frac{X_{0} U_{1}}{\sigma^{\prime \prime}}, \\
Y_{3}=X_{2}-\frac{3}{2} \frac{X_{0} U_{2}+X_{1} U_{1}}{\sigma^{\prime \prime}}+\frac{15}{8} \frac{X_{0} U_{1}^{2}}{\sigma^{\prime \prime 2}}, \\
Y_{4}=X_{3}-\frac{3}{2} \frac{X_{0} U_{3}+X_{1} U_{2}+X_{2} U_{1}}{\sigma^{\prime \prime}}+\frac{15}{8} \frac{2 X_{0} U_{1} U_{2}+X_{1} U_{1}^{2}}{\sigma^{\prime \prime 2}} \\
-\frac{35}{16} \frac{X_{0}}{\sigma^{\prime \prime 3}} .
\end{gathered}
$$

Once the perturbative terms $U_{n}(\sigma)$ are known it is straightforward to calculate the energy coefficients $e_{n}^{\text {best }}, n \geqslant 1$ from the virial energy (A15)

$$
e_{n}^{\text {best }}=-\frac{1}{\sqrt{\pi}} \int_{0}^{\infty} d \sigma \frac{\exp (-\sigma)}{\sqrt{\sigma}}\left(\frac{3}{2}-\sigma\right) \epsilon_{n}(\sigma)
$$

with (again suppressing the argument $\sigma$ )

$$
\begin{gathered}
\epsilon_{1}=1, \quad \epsilon_{2}=-\frac{1}{2} \frac{U_{1}}{\sigma}, \quad \epsilon_{3}=-\frac{1}{2} \frac{U_{2}}{\sigma}+\frac{3}{8} \frac{U_{1}^{2}}{\sigma^{2}}, \\
\epsilon_{4}=-\frac{1}{2} \frac{U_{3}}{\sigma}+\frac{3}{4} \frac{U_{1} U_{2}}{\sigma^{2}}-\frac{5}{16} \frac{U_{1}^{3}}{\sigma^{3}},
\end{gathered}
$$

$$
\epsilon_{5}=-\frac{1}{2} \frac{U_{4}}{\sigma}+\frac{3}{8} \frac{2 U_{1} U_{3}+U_{2}^{2}}{\sigma^{2}}-\frac{15}{16} \frac{U_{1}^{2} U_{2}}{\sigma^{3}}+\frac{35}{128} \frac{U_{1}^{4}}{\sigma^{4}} .
$$


We have evaluated Eqs. (A25)-(A32) by numerical integration. This is a nontrivial task because of the square-root singularities at $\sigma=0$ and the nonanalytic behavior of $\mu^{2}(\mid \sigma$ $\left.-\sigma^{\prime} \mid\right)$. The first problem was solved by transforming to $\sigma$ $=s^{2}, \sigma^{\prime}=s^{\prime 2}$, etc., the second one by using the trapezoidal integration rule so that $s=s^{\prime}$ is precisely hit (and not integrated over). In addition, for the first three intervals of each integral a Newton-Cotes formula of open type [Eq. (25.4.21) in Ref. [47]] was employed in order to avoid evaluation of the various integrands at $s=0$. While this cures the integrable singularities at the origin, it makes the treatment of the delay more problematic: in general $U_{n}\left(\left|\sigma \pm \sigma^{\prime}\right|=\left|s^{2} \pm s^{\prime 2}\right|\right)$ is not in the tabulated values of $U_{n}\left(\sigma=s^{2}\right)$ so that a three-term interpolation had to be used. In addition, the values of $U_{n>1}$ for small $\sigma$ were determined from the $(\sigma=0)$ limit of Eqs. (A22) and (A23)

$$
\ddot{\mu}^{2}(0)=-\frac{2 \alpha}{3 \sqrt{\pi}} \int_{0}^{\infty} d \sigma \frac{\exp (-\sigma)}{\left[\mu_{\text {best }}^{2}(\sigma)\right]^{1 / 2}},
$$

i.e.,

$$
\begin{gathered}
U_{n}(\sigma) \stackrel{\sigma \rightarrow 0}{\longrightarrow}\left(-\frac{1}{3 \sqrt{\pi}} \int_{0}^{\infty} d \sigma \frac{\exp (-\sigma)}{\sqrt{\sigma}} \epsilon_{n}(\sigma)\right) \sigma^{2}+\cdots, \\
n \geqslant 1
\end{gathered}
$$

with the same functions $\epsilon_{n}(\sigma)$ as used for calculating the energy coefficients.

Although the trapezoidal (as well as the Newton-Cotes) integration rule is not very precise [it exhibits errors of $O\left(h^{3}\right)$ where $h=s_{\max } / N$ is the increment] it offers an additional advantage: the tabulation of $U_{n}\left(\sigma=s^{2}\right)$ could be done step by step avoiding the time-consuming calculation of the integral over $\sigma^{\prime}$ in Eq. (A24) for each value of $\sigma$. Taking $\sigma_{\max }=20$ so that the retardation factor $\exp (-\sigma)$ is sufficiently small at the upper limit of integration, we have achieved stable numerical results with $N=1000-1500$. The numerical value of the second-order coefficient (A21) was confirmed with high accuracy (seven digits).

\section{APPENDIX B: ANALYTICAL RESULTS FOR THE CUMULANTS $\lambda_{1}$ AND $\lambda_{2}$}

Here we calculate the cumulants $\lambda_{n}$ for $n=1,2$. In the first case $a_{11}=t-t^{\prime} \equiv \sigma$ and we have for the first moment

$$
\begin{aligned}
m_{1} & =\frac{-\alpha}{\sqrt{4 \pi}} \int_{0}^{\beta} d t \int_{0}^{t} d t^{\prime} \int_{0}^{\infty} d u \frac{\exp \left[-\left(t-t^{\prime}\right)\right]}{\left(t-t^{\prime}+u\right)^{3 / 2}} \\
& =\frac{-\alpha}{\sqrt{4 \pi}} \int_{0}^{\beta} d t \int_{0}^{t} d t^{\prime} \exp \left[-\left(t-t^{\prime}\right)\right] \frac{2}{\sqrt{t-t^{\prime}}} \\
& =-\frac{\alpha}{\sqrt{\pi}} \int_{0}^{\beta} d \sigma \int_{\sigma / 2}^{\beta-\sigma / 2} d \Sigma \frac{\exp (-\sigma)}{\sqrt{\sigma}} \\
& =-\frac{\alpha}{\sqrt{\pi}} \int_{0}^{\beta} d \sigma(\beta-\sigma) \frac{\exp (-\sigma)}{\sqrt{\sigma}} .
\end{aligned}
$$

The remaining $\sigma$ integration is easily done by substituting $s=\sigma^{2}$. This gives

$$
\begin{aligned}
\lambda_{1} \equiv & m_{1}=-\alpha\left[\left(\beta-\frac{1}{2}\right) \operatorname{erf}(\sqrt{\beta})+\sqrt{\frac{\beta}{\pi}} e^{-\beta}\right] \\
& \stackrel{\beta \rightarrow \infty}{\rightarrow}-\alpha\left[\beta-\frac{1}{2}+\frac{1}{\sqrt{\beta \pi}} e^{-\beta}+\cdots\right],
\end{aligned}
$$

where $\operatorname{erf}(x)$ is the error function [47]. Thus we indeed have $e_{1}=-1$ for the first-order coefficient of the expansion of the ground-state energy in powers of the coupling constant. It is also seen that the subleading term in $\lambda_{1}$ is a constant which disappears if one calculates the derivative of the cumulant with respect to $\beta$ :

$$
\frac{\partial \lambda_{1}(\beta)}{\partial \beta}=-\alpha \operatorname{erf}(\sqrt{\beta}) \stackrel{\beta \rightarrow \infty}{\rightarrow}-\alpha\left[1-\frac{1}{\sqrt{\beta \pi}} e^{-\beta}+\cdots\right] .
$$

The analytical calculation is more involved for $n=2$. We start from Eq. (72) for the derivative of the second cumulant and substitute $S=\Sigma_{1}-\Sigma_{2}$ for the integration variable $\Sigma_{1,2}$ with $\Sigma_{2,1}=\beta-\sigma_{2,1} / 2$ fixed. This gives

$$
\begin{aligned}
\frac{\partial \lambda_{2}}{\partial \beta}= & \frac{4 \alpha^{2}}{\pi} \int_{0}^{\beta} d \sigma_{1} \int_{0}^{\sigma_{1}} d \sigma_{2} \frac{\exp \left(-\sigma_{1}-\sigma_{2}\right)}{\sqrt{\sigma_{1} \sigma_{2}}} \\
& \times \int_{0}^{\beta-s} d S f_{2}\left(\frac{a_{12}}{\sqrt{\sigma_{1} \sigma_{2}}}\right),
\end{aligned}
$$

where $s=\left(\sigma_{1}+\sigma_{2}\right) / 2 \geqslant r=\left(\sigma_{1}-\sigma_{2}\right) / 2 \geqslant 0$. The explicit form (49) of $a_{12}$ may now be used to write the last integral in Eq. (B4) as

$$
\int_{0}^{r} d S f_{2}\left(\frac{s-r}{\sqrt{\sigma_{1} \sigma_{2}}}\right)+\int_{r}^{\min (s, \beta-2)} d S f_{2}\left(\frac{s-S}{\sqrt{\sigma_{1} \sigma_{2}}}\right),
$$

where the two parts correspond to the constant and linear behavior of $a_{12}$, respectively, on the $(S \geqslant 0)$ side of Fig. 1 . We thus obtain

$$
\begin{aligned}
\frac{\partial \lambda_{2}}{\partial \beta}= & \frac{4 \alpha^{2}}{\pi} \int_{0}^{\beta} d \sigma_{1} \int_{0}^{\sigma_{1}} d \sigma_{2} e^{-\sigma_{1}-\sigma_{2}} \\
& \times\left\{\frac{\sigma_{1}-\sigma_{2}}{\sqrt{\sigma_{1} \sigma_{2}}} f_{2}\left(\sqrt{\frac{\sigma_{2}}{\sigma_{1}}}\right)+2 \int_{t_{0}}^{\sqrt{\sigma_{2} / \sigma_{1}}} d t f_{2}(t)\right\}
\end{aligned}
$$

with

$$
t_{0}\left(\sigma_{1}, \sigma_{2}, \beta\right)=\frac{\sigma_{1}+\sigma_{2}-\beta}{\sqrt{\sigma_{1} \sigma_{2}}} \Theta\left(\sigma_{1}+\sigma_{2}-\beta\right) .
$$

One sees that for $\beta \rightarrow \infty t_{0}\left(\sigma_{1}, \sigma_{2}, \beta\right) \rightarrow 0$ since the relative times are bounded by the exponential retardation factors. In other words, 


$$
\begin{aligned}
\frac{\partial \lambda_{2}}{\partial \beta} \rightarrow & \frac{2 \alpha^{2}}{\pi} \int_{0}^{\infty} d \sigma_{1} e^{-\sigma_{1}} \int_{0}^{\sigma_{1}} d \sigma_{2} e^{-\sigma_{2}} \\
& \times\left\{\frac{\sigma_{1}-\sigma_{2}}{\sqrt{\sigma_{2} \sigma_{1}}} f_{2}\left(\sqrt{\frac{\sigma_{2}}{\sigma_{1}}}\right)+2 \int_{0}^{\sqrt{\sigma_{2} / \sigma_{1}}} d t f_{2}(t)\right\}
\end{aligned}
$$

and the corrections are of order $\exp (-\beta)$. Putting $\sigma_{2}=t^{2} \sigma_{1}$, the $\sigma_{1}$ integration can be performed in the first term and an integration by parts in the second term gives

$$
\frac{\partial \lambda_{2}}{\partial \beta} \rightarrow \frac{4 \alpha^{2}}{\pi} \int_{0}^{1} d t\left[\frac{\arcsin (t)}{t}-1\right]\left[\frac{2}{\left(1+t^{2}\right)^{2}}-\frac{1}{2}\right]+O\left(e^{-\beta}\right) .
$$

Finally a combination of partial integrations [to get rid of the $\arcsin (t)]$ and integrals which MAPLE can do, leads to

$$
\frac{\partial \lambda_{2}}{\partial \beta} \rightarrow \alpha^{2}[4 \ln (1+\sqrt{2})-3 \ln 2-\sqrt{2}]+O\left(e^{-\beta}\right) .
$$

We thus obtain the second-order coefficient of the groundstate energy as given in Eq. (7).

In our approach it is very important to know the precise way how Eq. (B10) approaches the asymptotic value calculated above. The easiest way to find out is to differentiate Eq. (B6) again with respect to $\beta$ :

$$
\begin{aligned}
\frac{\partial^{2} \lambda_{2}}{\partial \beta^{2}}= & \frac{2 \alpha^{2}}{\pi} e^{-\beta} \int_{0}^{\beta} d \sigma_{2} e^{-\sigma_{2}} \\
& \times\left\{\frac{\beta-\sigma_{2}}{\sqrt{\beta \sigma_{2}}} f_{2}\left(\sqrt{\frac{\sigma_{2}}{\beta}}\right)+2 \int_{t_{0}\left(\beta, \sigma_{2}, \beta\right)}^{\sqrt{\sigma_{2} / \beta}} d t f_{2}(t)\right\} \\
& -\frac{4 \alpha^{2}}{\pi} \int_{0}^{\beta} d \sigma_{1} e^{-\sigma_{1}} \int_{0}^{\sigma_{1}} d \sigma_{2} e^{-\sigma_{2}} f_{2}\left(t_{0}\right) \\
& \times \frac{\partial t_{0}\left(\sigma_{1}, \sigma_{2}, \beta\right)}{\partial \beta}=: I_{1}+I_{2} .
\end{aligned}
$$

Consider first the contribution $I_{1}$ : since $t_{0}\left(\beta, \sigma_{2}, \beta\right)=\sqrt{\sigma_{2} / \beta}$ is the same as the upper limit of the integral, the latter vanishes so that

$$
I_{1}=\frac{2 \alpha^{2}}{\pi} e^{-\beta} \int_{0}^{\beta} d \sigma_{2} e^{-\sigma_{2}} \frac{\beta-\sigma_{2}}{\sqrt{\beta \sigma_{2}}} f_{2}\left(\sqrt{\frac{\sigma_{2}}{\beta}}\right) .
$$

The substitution $\sigma_{2}=\beta s^{2}$ gives

$$
I_{1}=\frac{4 \alpha^{2}}{\pi} e^{-\beta} \beta \int_{0}^{1} d s e^{-\beta s^{2}}\left(1-s^{2}\right)\left[\frac{\arcsin s}{s}-1\right]
$$

and in the limit $\beta \rightarrow \infty$ the exponential factor forces $s \rightarrow 0$ in all other terms [48]. Therefore we may expand these in powers of $s$, integrate term by term, and obtain

$$
\begin{aligned}
I_{1} & \rightarrow \frac{4 \alpha^{2}}{\pi} e^{-\beta} \beta \int_{0}^{1} d s e^{-\beta s^{2}}\left[\frac{1}{6} s^{2}-\frac{11}{120} s^{4}+\cdots\right] \\
& =\frac{\alpha^{2}}{6 \sqrt{\pi}} \frac{\exp (-\beta)}{\sqrt{\beta}}\left[1+O\left(\frac{1}{\beta}\right)\right]
\end{aligned}
$$

For the contribution $I_{2}$ we use

$$
\frac{\partial t_{0}}{\partial \beta}=-\frac{\Theta\left(\sigma_{1}+\sigma_{2}-\beta\right)}{\sqrt{\sigma_{1} \sigma_{2}}}
$$

so that

$$
\begin{aligned}
I_{2}= & \frac{4 \alpha^{2}}{\pi} \int_{0}^{\beta} d \sigma_{1} \int_{0}^{\sigma_{1}} d \sigma_{2} \frac{\Theta\left(\sigma_{1}+\sigma_{2}-\beta\right)}{\sqrt{\sigma_{1} \sigma_{2}}} \\
& \times e^{-\sigma_{1}-\sigma_{2}} f_{2}\left(\frac{\sigma_{1}+\sigma_{2}-\beta}{\sqrt{\sigma_{1} \sigma_{2}}}\right) .
\end{aligned}
$$

Using the variables of Eq. (48) we obtain

$$
I_{2}=\frac{4 \alpha^{2}}{\pi} 2 \int_{\beta / 2}^{\beta} d s \int_{0}^{\beta-s} d r \frac{\exp (-2 s)}{\sqrt{s^{2}-r^{2}}} f_{2}\left(\frac{2 s-\beta}{\sqrt{s^{2}-r^{2}}}\right)
$$

since the Jacobian of the transformation is 2 . The substitutions $s=\beta(1+u) / 2, r=s \sin \phi$ give

$$
I_{2}=\frac{4 \alpha^{2}}{\pi} \beta \beta e^{-\beta} \int_{0}^{1} d u e^{-\beta u} \int_{0}^{\phi_{0}(u)} d \phi f_{2}\left(\frac{2 u}{(1+u) \cos \phi}\right),
$$

where $\sin \phi_{0}(u)=(1-u) /(1+u)$. Again, for $\beta \rightarrow \infty$ the low- $u$ behavior of the nonexponential part of the integrand determines the asymptotic behavior. We have

$$
\begin{aligned}
g(u):= & \int_{0}^{\phi_{0}(u)} d \phi f_{2}\left(\frac{2 u}{1+u} \frac{1}{\cos \phi}\right) \\
\rightarrow & \frac{1}{6}\left(\frac{2 u}{1+u}\right)^{2} \int_{0}^{\phi_{0}(u)} d \phi \frac{1}{\cos ^{2} \phi} \\
& +\frac{3}{40}\left(\frac{2 u}{1+u}\right)^{4} \int_{0}^{\phi_{0}(u)} d \phi \frac{1}{\cos ^{4} \phi}+\cdots .
\end{aligned}
$$

The $\phi$ integrals are elementary (see, e.g., Ref. [29], pp. 103, 104) and one obtains

$$
g(u) \stackrel{u \rightarrow 0}{\rightarrow} \frac{1}{3} u^{3 / 2}+O\left(u^{5 / 2}\right) .
$$

Therefore

$$
I_{2} \rightarrow \frac{\alpha^{2}}{\sqrt{\pi}} \frac{\exp (-\beta)}{\beta^{3 / 2}}
$$

is subasymptotic and after integration of Eq. (B14) with respect to (large) $\beta$ we obtain 


$$
e_{2}(\beta) \stackrel{\beta \rightarrow \infty}{\rightarrow} e_{2}-\frac{1}{12 \sqrt{\pi}}=\frac{\exp (-\beta)}{\beta^{1 / 2}} .
$$

Comparison with Eq. (B3) shows that this is the same functional approach to the asymptotic value as for the case $n=1$; only the numerical coefficient is different.

\section{APPENDIX C: TANH-SINH INTEGRATION}

Here we briefly outline the "tanh-sinh-integration" procedure proposed by Takahashi and Mori [33] and used in most of our deterministic calculations. For a one-dimensional integral over the interval $x \in[-1,+1]$ it is based on the transformation

$$
\begin{gathered}
x=g(t)=\tanh (\kappa \sinh t), \quad t \in[-\infty,+\infty], \\
g^{\prime}(t)=\frac{1}{\cosh ^{2}(\kappa \sinh t)} \kappa \cosh t
\end{gathered}
$$

which has the effect that the transformed integrand $g^{\prime}(t) f[g(t)]$ vanishes at the boundaries along with all derivatives [for sufficiently well-behaved $f(x)$ ]. Therefore the Euler-Maclaurin summation formula [see, e.g., Ref. [47], Eq. (25.4.7)] with a stepsize $h$ does not get any (power) contributions from the endpoints and we have

$$
\int_{-1}^{+1} d x f(x)=\int_{-\infty}^{+\infty} d t g^{\prime}(t) f[g(t)] \approx h \sum_{k=-\infty}^{k=+\infty} w_{k} f\left(x_{k}\right)
$$

with

$$
x_{k}=g(k h) \equiv \tanh [\kappa \sinh (k h)] \text {, }
$$

$$
w_{k}=g^{\prime}(k h) \equiv \frac{1}{\cosh ^{2}[\kappa \sinh (k h)]} \kappa \cosh (k h) .
$$

For large $|k|$ and fixed $h$ we find

$$
\begin{gathered}
x_{k} \rightarrow 1-2 \exp \left(-\kappa e^{|k| h}\right), \\
w_{k} \rightarrow 2 \kappa \exp \left(-\kappa e^{|k| h}+|k| h\right)
\end{gathered}
$$

showing the "double-exponential" character of this transformation.

Although the value $\kappa=\pi / 2$ has been reported to be optimal [35] we have found little difference in efficiency by taking

$$
\kappa=1,
$$

which is our choice in this work. In practice, the infinite sum in Eq. (C3) is finite since the weights $w_{k}$ decrease rapidly with $|k|$ as seen in Eq. (C7). We use

$$
h|k| \leqslant h k_{\max }=3.4
$$

as a cutoff so that $x_{ \pm k \max }= \pm\left(1-2.01 \times 10^{-13}\right)$ and $w_{ \pm k \max }$ $=6.02 \times 10^{-12}$. The number of function calls then is

$$
n_{t}=2 k_{\max }+1 \text {. }
$$

Conversely, if $n_{t}$ is chosen (as we do to estimate the run time in advance) the increment is given by

$$
h=\frac{6.8}{n_{t}-1} .
$$

It is straightforward to extend Eq. (C3) to an arbitrary integral as shown in Eq. (80) in the main text.
[1] D. Hanneke, S. Fogwell, and G. Gabrielse, Phys. Rev. Lett. 100, 120801 (2008).

[2] B. C. Odom, D. Hanneke, B. D’Urso, and G. Gabrielse, Phys. Rev. Lett. 97, 030801 (2006).

[3] G. Gabrielse, D. Hanneke, T. Kinoshita, M. Nio, and B. C. Odom, Phys. Rev. Lett. 97, 030802 (2006); 99, 039902(E) (2007).

[4] C. Itzykson and J.-B. Zuber, Quantum Field Theory (McGrawHill, New York, 1980), Eq. (9-195).

[5] S. Laporta and E. Remiddi, Phys. Lett. B 379, 283 (1996).

[6] T. Aoyama, M. Hayakawa, T. Kinoshita, and M. Nio, Phys. Rev. D 77, 053012 (2008); Phys. Rev. Lett. 99, 110406 (2007); T. Kinoshita and M. Nio, Phys. Rev. D 73, 013003 (2006).

[7] T. Kinoshita and M. Nio, Phys. Rev. D 73, 053007 (2006).

[8] N. N. Bogolyubov, Jr. and V. N. Plechko, Theor. Math. Phys. 65, 1255 (1985).

[9] R. Rosenfelder and A. W. Schreiber, Phys. Rev. D 53, 3337 (1996).

[10] R. Rosenfelder and A. W. Schreiber, Phys. Rev. D 53, 3354 (1996).

[11] C. Alexandrou, R. Rosenfelder, and A. W. Schreiber, Phys. Rev. A 59, 1762 (1999).
[12] C. Alexandrou, R. Rosenfelder, and A. W. Schreiber, Phys. Rev. D 62, 085009 (2000).

[13] R. Rosenfelder, in Path Integrals - New Trends and Perspectives, Proceedings of the 9th International Conference, Dresden, Germany, 2007 edited by W. Janke and A. Pelster (World Scientific, Singapore, 2008).

[14] One could also consider the perturbative expansion of the effective mass $m^{\star}(\alpha)$.

[15] G. Höhler and A. Müllensiefen, Z. Phys. 157, 159 (1959); J. Röseler, Phys. Status Solidi 25, 311 (1968).

[16] M. A. Smondyrev, Theor. Math. Phys. 68, 653 (1986).

[17] O. V. Selyugin and M. A. Smondyrev, Phys. Status Solidi B 155, 155 (1989).

[18] R. P. Feynman, Phys. Rev. 97, 660 (1955).

[19] Except when $\sigma$ is close to $\beta$ which, however, is suppressed dynamically by a large spatial difference.

[20] J. Adamowski, B. Gerlach, and H. Leschke, in Functional Integration-Theory and Application, edited by J. P. Antoine and E. Tirapegui (Plenum, New York, 1980), p. 291; M. Saitoh, J. Phys. Soc. Jpn. 49, 878 (1980).

[21] R. Rosenfelder, J. Phys. A 27, 3523 (1994).

[22] R. Rosenfelder, Ann. Phys. 128, 188 (1980).

[23] I. S. Gradshteyn and I. M. Ryzhik, Table of Integrals, Series 
and Products, 4th ed. (Academic Press, New York, 1980).

[24] Zero eigenvalues may occur as function of the various times but such that the corresponding integrals still exist. Therefore, in general, $(A)$ is only positive semidefinite.

[25] R. A. Horn and C. R. Johnson, Matrix Analysis (Cambridge University Press, Cambridge, 1985), Chap. 7.

[26] O. Hotz and B. Sturmfels, e-print arXiv:0604374v2.

[27] W. H. Greub, Linear Algebra, 2nd ed. (Springer, Berlin, 1963), p. 440.

[28] Contrary to the mathematical nomenclature we characterize the principal minors by the index of the row +column which has been cut out.

[29] H. B. Dwight, Tables of Integrals and Other Mathematical Data (MacMillan, New York, 1961).

[30] This is familiar from time-dependent perturbation theory in quantum mechanics, see, e.g., A. L. Fetter and J. D. Walecka, Quantum Theory of Many-Particle Systems (McGraw-Hill, New York, 1971), pp. 57, 58.

[31] G. P. Lepage, J. Comput. Phys. 27, 192 (1978); G. P. Lepage (unpublished).

[32] This may be balanced by a better fit to the remaining data points in which case an unambigous determination of $e_{n}$ is not possible.

[33] H. Takahashi and M. Mori, Publ. Res. Inst. Math. Sci. 9, 721 (1974).

[34] J. Borwein, D. Bailey, and R. Girgensohn, Experimentation in Mathemathics: Computational Paths to Discovery (A.K. Peters, Natick, MA, 2004), Chap. 7.4.3; D. H. Bailey, J. M. Borwein, D. Broadhurst, and M. L. Glasser, J. Phys. A 41, 205203 (2008).

[35] K. Murota and M. Iri, Numer. Math. 38, 347 (1982).
[36] F. Stenger, Numerical Methods Based on Sinc and Analytic Functions (Springer, New York, 1993), Chap. 4.2.

[37] With only $10^{8}$ Monte Carlo calls and $n_{t}=21$, i.e., $n_{\text {tot }}=2$ $\times 10^{9}$ (one order of magnitude less function calls than for the data of Table I) one obtains $e_{3}=-0.8061(1) \times 10^{-3}$ [one order of magnitude more precise than given in Eq. (77)].

[38] T. Hahn, Comput. Phys. Commun. 168, 78 (2005); Nucl. Instrum. Methods Phys. Res. A 559, 273 (2006).

[39] T. Kinoshita and M. Nio, Phys. Rev. D 70, 113001 (2004).

[40] W.-F. Lu, S. K. You, J. Bak, C. K. Kim, and K. Nahm, J. Phys. A 35, 21 (2002).

[41] This is probably due to an arithmetic error in the fourth cumulant as Eq. (26) in that paper does not have a proper $\beta \rightarrow \infty$ limit while the lower cumulants in Eqs. (24) and (25) do.

[42] B. Gerlach and H. Löwen, Rev. Mod. Phys. 63, 63 (1991).

[43] R. Rosenfelder and A. W. Schreiber, Phys. Lett. A 284, 63 (2001).

[44] It is also the mean value of $\left[\mathbf{x}(t)-\mathbf{x}\left(t^{\prime}\right)\right]^{2}$ with respect to the trial action for $\beta \rightarrow \infty$ which suggests a more physical interpretation [46].

[45] Similar integrals have been evaluated in Appendix B of $\mathrm{J}$. Devreese, J. de Sitter, and M. Goovaerts, Phys. Rev. B 5, 2367 (1972).

[46] R. Rosenfelder and A. W. Schreiber, Eur. Phys. J. C 37, 161 (2004).

[47] Handbook of Mathematical Functions, edited by M. Abramowitz and I. Stegun (Dover, New York, 1965).

[48] This is just Laplace's method [see, e.g., L. Sirovich, Techniques of Asymptotic Analysis (Springer, New York, 1971), p. $80]$ for the asymptotic evaluation of euclidean integrals. 